Proceedings of the Edinburgh Mathematical Society (2005) 48, 389-421 (C)

DOI:10.1017/S0013091503000890 Printed in the United Kingdom

\title{
SOME DUALITY CONJECTURES FOR FINITE GRAPHS AND THEIR GROUP THEORETIC CONSEQUENCES
}

\author{
S. M. GERSTEN ${ }^{1}$ AND T. R. RILEY ${ }^{2}$ \\ ${ }^{1}$ Mathematics Department, 155 S 1400E, Room 233, University of Utah, \\ Salt Lake City, UT 84112, USA (gersten@math.utah.edu) \\ ${ }^{2}$ Department of Mathematics, Yale University, 10 Hillhouse Avenue, \\ PO Box 208283, New Haven, CT 06520-8283, USA (tim.riley@yale.edu)
}

(Received 22 October 2003)

\begin{abstract}
We pose some graph theoretic conjectures about duality and the diameter of maximal trees in planar graphs, and we give innovations in the following two topics in geometric group theory, where the conjectures have applications.

Central extensions. We describe an electrostatic model concerning how van Kampen diagrams change when one takes a central extensipn of a group. Mpdulo the conjectures, this leads to a new proof that finitely generated class $c$ nilpptent groups admit degree $c+$ pol nomial isopdrimetric functions.

Filling functions. We collate and exesults abolut incerre ationships betwen filling functions for finite presentations of g.oups. We use the electrostatic prodpl in proving that the gallery length filling function, which measures the diameter of the duals of diaglams, is qualitatively the same as a filling function D $\log \mathrm{A}$, concernihg the sum of the diameter w wh thd ldgarith $\mathrm{m}$ of the area of a diagram. We show that the conjectures imply that the space-complexity filling function filling length essentially equates to gallery length. We give linear upper bounds on these functions for a number of classes of groups including fundamental groups of compact geometrizable 3-manifolds, certain graphs of groups, and almost convex groups. Also we define restricted filling functions which concern diagrams with uniformly bounded vertex valence, and we show that, assuming the conjectures, they reduce to just two filling functions - the analogues of non-deterministic space and time.
\end{abstract}

Keywords: isoperimetric function; gallery length; van Kampen diagram; central extension; planar graph; duality

2000 Mathematics subject classification: Primary 20F05; 20F06; 57M05; 57M20

\section{Introduction}

We begin by presenting one of the graph theoretic conjectures originating in our work in geometric group theory.

Suppose $G$ is a finite, connected, undirected graph* embedded $\dagger$ in the 2 -sphere (whence

* We allow graphs to have multiple edges between two vertices and to have edges that meet only one vertex, thereby forming a loop. Thus $G$ may be what is referred to as a multigraph in [7].

$\dagger$ To avoid pathologies we assume here, and elsewhere without further comment, that each edge is embedded as a concatenation of finitely many geodesic arcs. 
$G$ is planar). Let $G^{\star}$ be the dual graph to $G$ (see $\S 4$ ). Given a maximal* tree $T$ in $G$, define $T^{\star}$ to be the subgraph of $G^{\star}$ made up of edges dual to edges in $G \backslash T$. Refer to $\left(T, T^{\star}\right)$ as a complementary pair of maximal trees on account of the following (easy) lemma.

Lemma 1.1. The graph $T^{\star}$ is a maximal tree in $G^{\star}$.

Figure 1 in $\S 3$ shows two examples of complementary pairs $\left(T, T^{\star}\right)$ in a graph $G$. The tree $T$ is drawn with heavy lines and $T^{\star}$ with dotted lines.

We endow $G$ with the combinatorial metric in which each edge has length 1 . The diameter $\operatorname{Diam}(G)$ is the maximum distance between pairs of vertices of the graph. It is not hard to construct a maximal tree $T$ in a connected graph $G$ in such a way that $T$ has diameter closely related to the diameter of $G$. Specifically, choose a base vertex $v_{0}$ in $G$ and take $T$ to be a maximal geodesic tree based at $v_{0}$. Then $\operatorname{Diam}(G) \leqslant \operatorname{Diam}(T) \leqslant$ $2 \operatorname{Diam}(G)$.

Our concern is with the existence of a complementary pair of trees $\left(T, T^{\star}\right)$ that both have similarly controlled diameters. The first of our conjectures is below. Others are listed in $\S 2$, followed by some examples in $\S 3$ and then a reformulation in the language of diagrams in $\S 4$.

Conjecture 1.2. Fix any $\lambda>0$. There exists some constant $K>0$, depending only on $\lambda$, with the following property. Suppose that $G$ is a/finite, connected graph embedded in the 2-sphere, and that the nalencet of each nertex $1 n$ c ${ }^{\star}$ is at most $\lambda$. Then there is a maximal tree $T$ in $G$ with

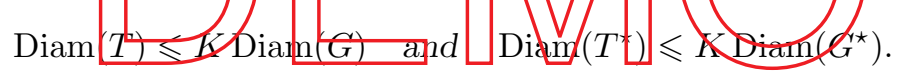

A reason we became interested in these questions is that if true they imply the following, whose applications we will explain.

Conjecture 5.4. The filling functions $\mathrm{GL}_{\mathcal{P}}$ and $\mathrm{DGL}_{\mathcal{P}}$ for any given finite group presentation $\mathcal{P}$ are $\simeq$-equivalent.

This says that a filling function $\mathrm{DGL}_{\mathcal{P}}$ for finite presentations of groups reduces to a simpler filling function $\mathrm{GL}_{\mathcal{P}}$ called gallery length. Careful definitions of these terms can be found in $\S 5$; what follows is a brief overview.

Let $\mathcal{P}$ be a finite presentation of a group $\Gamma$. The word problem for $\mathcal{P}$, as posed by Dehn $[6]$ towards the beginning of the last century, asks for a systematic method (since interpreted as an algorithm) to determine, given a word $w$ in the generators, whether or not $w$ represents 1 in $\Gamma$. This began a rich seam in combinatorial and geometric group theory, remarkably involving not only issues of undecidability and algorithmic complexity but also geometry and topology (illuminated most vividly by Gromov in [14]).

\footnotetext{
* A maximal tree (also known as a spanning tree) in a connected graph $G$ is a subtree such that, if one included any further edge of $G$, then the resulting graph would no longer be a tree.

$\dagger$ The valence $\lambda$ of a vertex $v$ in $G$ is the number of connected components of $(G \backslash v) \cap B_{v}(\varepsilon)$, the intersection of $G \backslash v$ with a small neighbourhood of $v$. Equivalently, $\lambda$ is the length of the boundary circuit of the face $v^{\star}$ dual to $v$. (This is different from the number of edges in the boundary of the face in the event that there is an edge in $G$ that forms a loop based at $v$.)
} 
Filling functions $\mathbb{N} \rightarrow \mathbb{N}$ capture aspects of the geometry of the word problem for a presentation $\mathcal{P}$ of a group $\Gamma$. They concern van Kampen diagrams, which are connected, planar 2-complexes that provide graphical demonstrations of how words $w$ that represent the identity in $\Gamma$ are consequences of the defining relations in $\mathcal{P}$. Filling functions arise from measuring different aspects of the geometry of van Kampen diagrams. The most well known is $\operatorname{Area}_{\mathcal{P}}(n)$ and is referred to as the Dehn function or minimal isoperimetric function for $\mathcal{P}$. It is the minimum number $K$ such that all words $w$ of length at most $n$ that represent 1 in $\Gamma$ admit a van Kampen diagram with at most $K 2$-cells. The 1 -skeleton $G$ of a van Kampen diagram $D$ is a finite, planar graph and so falls within the scope of our conjectures. The gallery length filling function $\mathrm{GL}_{\mathcal{P}}(n)$ measures the diameter of $G^{\star}$. And $\operatorname{DGL}_{\mathcal{P}}(n)$ measures the minimal value of $\operatorname{Diam}(T)+\operatorname{Diam}\left(T^{\star}\right)$ ranging over all complementary pairs of maximal trees for $G$.

If true, the graph theoretic conjectures would allow us to control $\operatorname{Diam}(T)$ in terms of $\operatorname{Diam}\left(T^{\star}\right)$. It would follow that $\operatorname{DGL}(n)$ is qualitatively the same function as $\operatorname{GL}(n)$, and that is the content of Conjecture 5.4.

A first application is set out in $\S 6$. It concerns central extensions $\hat{\Gamma}$ of groups $\Gamma$. We give an electrostatic model for obtaining van Kampen diagrams with respect to a finite presentation $\hat{\mathcal{P}}$ for $\hat{\Gamma}$ by blowing up van Kampen diagrams for words in a finite presentation $\mathcal{P}$ for $\Gamma$. An analysis of the change in the geometry of van Kampen diagrams

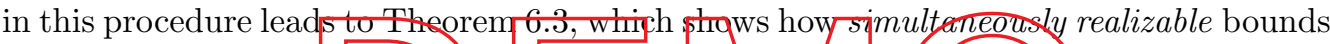

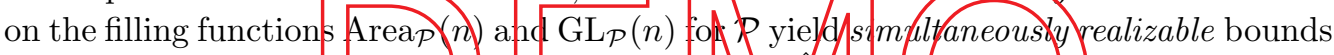

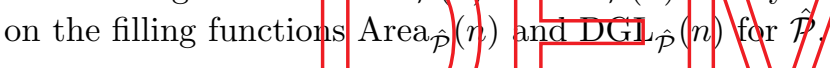

Theorem 6.3. Suppose that $1 \rightarrow \Lambda \rightarrow \hat{\Gamma} \rightarrow \Gamma \rightarrow 1$ is a central extension of the finitely presented group $\Gamma$, and that $(f, g)$ is an (Area, DGL)-parr for a finite presentation $\mathcal{P}$ of $\Gamma$.

(i) If $\Lambda=\mathbb{Z}$, then, up to a common multiplicative constant, $\left(f(n) g(n)+n^{2}, g(n)+n\right)$ is an (Area, GL)-pair for the finite presentation $\hat{P}$ of Proposition 6.1 for $\hat{\Gamma}$.

(ii) If $\Lambda=C_{q}$, a finite cyclic group of order $q$, then, up to a common multiplicative constant, $(f(n)+n, g(n)+n)$ is an (Area, GL)-pair for the finite presentation $\hat{P}$ of Proposition 6.2 for $\hat{\Gamma}$.

If Conjecture 5.4 is true, then we can simplify this theorem by replacing $\operatorname{DGL}_{\hat{\mathcal{P}}}(n)$ by $\mathrm{GL}_{\hat{\mathcal{P}}}(n)$. This would make the result applicable iteratively, and we would therefore be able to constrain both the Dehn function and the gallery length function as one takes successive central extensions. As a corollary we would reproduce the result of [13-15] that finitely generated nilpotent groups of class $c$ admit polynomial isoperimetric functions of degree $c+1$.

A second application concerns a filling function $\mathrm{FL}_{\mathcal{P}}: \mathbb{N} \rightarrow \mathbb{N}$ known as the filling length function of a finite presentation $\mathcal{P}$. The filling length of a diagram $D$ is the minimal upper bound on the length of the boundary curve in the course of a shelling (combinatorial nullhomotopy) of $D$ down to its base vertex. And $\operatorname{FL}_{\mathcal{P}}(w)$ for a word $w$ that represents 1 in the group presented by $\mathcal{P}$ is the minimum of $\mathrm{FL}(D)$ over all van Kampen diagrams 
$D$ for $w$. And then $\operatorname{FL}_{\mathcal{P}}(n)$ is the maximum of $\operatorname{FL}_{\mathcal{P}}(w)$ quantifying over all words $w$ of length at most $n$ that represent 1 in the group. It is possible to interpret $\mathrm{FL}_{\mathcal{P}}$ as the non-deterministic space-complexity of a naive approach to solving the word problem for $\mathcal{P}$ in which relators are applied exhaustively (see $\S 5$ or $[\mathbf{1 3}]$ for more details).

We proved in Theorem 7.1 of $[\mathbf{1 2}]$ that $\mathrm{FL}_{\mathcal{P}} \simeq \mathrm{DGL}_{\mathcal{P}}$ under the technical hypothesis that $\mathcal{P}$ is fat (Definition 5.5), and so these two filling functions are qualitatively the same. If $\mathrm{DGL}_{\mathcal{P}} \simeq \mathrm{GL}_{\mathcal{P}}$, then we deduce (relevant definitions are given in detail in $\S 5$ ) the following theorem.

Theorem 1.3. Let $\mathcal{P}$ be a finite fat presentation. Assuming Conjecture 5.4 holds, the filling functions $\mathrm{FL}_{\mathcal{P}}, \mathrm{GL}_{\mathcal{P}}: \mathbb{N} \rightarrow \mathbb{N}$ for $\mathcal{P}$ satisfy $\mathrm{FL}_{\mathcal{P}} \simeq \mathrm{GL}_{\mathcal{P}}$.

Thus Conjecture 5.4 allows us to re-express $\mathrm{FL}_{\mathcal{P}}$ (already a ubiquitous concept as it is both a space-complexity measure and a differential-geometric invariant controlling the length of curves in null-homotopies) in attractive and concise geometric/combinatorial terms as a measure of the diameter of the duals of the 1-skeleta of van Kampen diagrams.

The electrostatic model is used again in $\S 7$. We show that a filling function $D \log \mathrm{A}_{\mathcal{P}}$ that measures the sum of the diameter with the logarithm of the area of a diagram is qualitatively the same as $\mathrm{GL}_{\mathcal{P}}$.

Theorem 7.1. If $\mathcal{P}$ is a fat finite presentation, then its filling functions $\mathrm{GL}_{\mathcal{P}}$ and

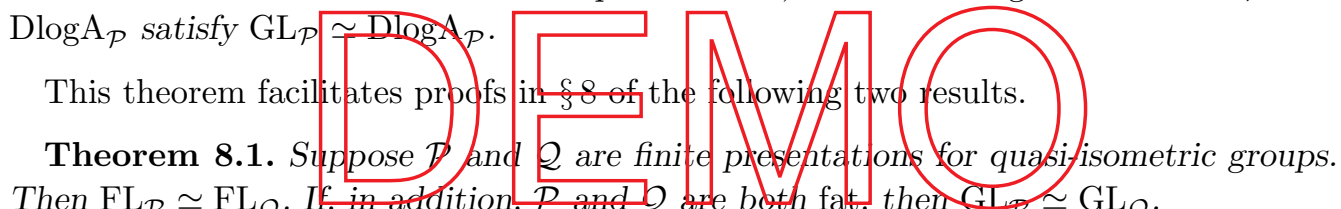

Theorem 8.2.

(i) The gallery length function of any finite presentation of a group admitting a polynomial isoperimetric inequality of degree $d \geqslant 2$ admits a polynomial upper bound of degree $d-1$.

(ii) The gallery length function of the presentation $\langle x, y, s, t|[x, y]=1, t x t^{-1}=$ $x^{2}$, sys $\left.^{-1}=y^{2}\right\rangle$, due to Bridson, admits a linear upper bound.

If Conjecture 5.4 holds, then the filling length functions admit the same upper bounds.

In $\S 8$ we also establish linear upper bounds on the filling length functions for a number of classes of groups.

Theorem 8.3. Asynchronously combable groups have filling length functions admitting linear upper bounds. This includes

(i) fundamental groups of finite graphs of groups with finitely generated free vertex and edge groups (for example, the Baumslag-Solitar group $\operatorname{BS}(p, q)=\langle x, y| y^{-1} x^{p} y=$ $\left.x^{q}\right\rangle$;

(ii) fundamental groups of compact, geometrizable 3-manifolds; 
(iii) split extensions of hyperbolic or abelian groups by asynchronously combable groups.

Theorem 8.4. The filling length function of any group $\Gamma$ that has a finite presentation $\mathcal{P}=\langle\mathcal{A} \mid \mathcal{R}\rangle$ satisfying Cannon's almost convexity condition $\mathrm{AC}(2)$ admits a linear upper bound.

And we ask questions about uniform bounds on the Dehn function and filling length function in larger classes of groups.

In $\S 9$ we collate the known results about the bounds that exist between filling functions for arbitrary finite presentations, and we explain the simplifying impact of Theorem 1.3 (modulo Conjecture 4.3 or 5.4).

In $\S 10$ we introduce and examine the theory of restricted filling functions. These concern measurements of van Kampen diagrams that have uniformly bounded vertex valences, i.e. diagrams whose curvature is uniformly bounded away from $-\infty$. In the course of the proof of Theorem 7.1 in $\S 7$ we show that every word that represents the identity in a fat presentation admits a van Kampen diagram in which every vertex has valence at most 12 . Moreover, we have considerable control on the geometry of this diagram. This enables us to understand how the restricted analogues of the filling functions interrelate. We set out the results in Theorem 10.3. If Conjecture 4.3 holds, then all the filling functions we consider collapse to just two: the (restricted analogues of) space and time complexity filling functrons if $\mathrm{L}_{\mathrm{p}}$ and Areat.

This is the second article ip a seeies that pegan with [121, in which we showed some of the ways in which duality considerations impact the study of diagrams and filling functions. We hope* to complete the series with ahird article contringing proofs of the conjectures in $\S 2$.

\section{The graph theoretic conjectures}

Four conjectures are set out below, using the notation established in $\S 1$. Two further conjectures appear in this paper. One, in $\S 4$, is phrased in the language of diagram measurements and the other, in $\S 5$, concerns filling functions. Proposition 2.5 summarizes the known interrelationships.

Conjecture 2.1. Fix any $\lambda>0$. There exists some constant $K>0$, depending only on $\lambda$, with the following property. Suppose that $G$ is a finite, connected graph embedded in the 2 -sphere, and that the valence of each vertex in $G^{\star}$ is at most $\lambda$. Then there is a maximal tree $T$ in $G$ with

$$
\operatorname{Diam}(T) \leqslant K \operatorname{Diam}(G) \text { and } \operatorname{Diam}\left(T^{\star}\right) \leqslant K \operatorname{Diam}\left(G^{\star}\right) .
$$

\footnotetext{
* Remark added August 2004. The conjectures remain resistant to resolution more than three years after a version was first publicized by the first author talking in the Workshop on Geometric Group Theory at the Centre de Recherches Mathématiques in Montreal in July 2001. Indeed, it is unknown whether the following statement, which is Conjecture 2.1 with reference to $\lambda$ removed, is true: there exists $K>0$ such that if $G$ is a finite, connected graph embedded in the 2-sphere, then there is a maximal tree $T$ in $G$ such that $\operatorname{Diam}(T) \leqslant K \operatorname{Diam}(G)$ and $\operatorname{Diam}\left(T^{\star}\right) \leqslant K \operatorname{Diam}\left(G^{\star}\right)$.
} 
Conjecture 2.2. Fix any $\lambda>0$. There exists some constant $K>0$ such that, with the same hypotheses as Conjecture 2.1, we can find a maximal tree $T$ in $G$ with

$$
\max \left\{\operatorname{Diam}(T), \operatorname{Diam}\left(T^{\star}\right)\right\} \leqslant K \operatorname{Diam}\left(G^{\star}\right)+K .
$$

Conjecture 2.3. The conclusion of Conjecture 2.1 holds if we additionally require the valence of every vertex in both $G$ and $G^{\star}$ to be at most $\lambda$.

Conjecture 2.4. Fix any $\lambda>0$. There exists some constant $K>0$, depending only on $\lambda$, with the following property. Suppose that $G$ is a finite, connected graph embedded in the 2 -sphere and that the valence of every vertex in $G^{\star}$ is at most $\lambda$ with the possible exception of one vertex $e_{\infty}^{\star}$. Define $n$ to be the valence of $e_{\infty}^{\star}$ in $G^{\star}$. Then there is a maximal tree $T$ in $G$ with

$$
\max \left\{\operatorname{Diam}(T), \operatorname{Diam}\left(T^{\star}\right)\right\} \leqslant K\left(\operatorname{Diam}\left(G^{\star}\right)+n\right) .
$$

Proposition 2.5. The following implications between the conjectures hold.

$$
2.1 \Rightarrow 2.2 \Rightarrow 2.3
$$

$\Uparrow$

Proof. If $G$ is a $\$ r$ raph as per Cenjecture 2.1. then we can find a path in $G$ between given vertices $a$ and $b$ by takjing $\bar{a}$ and $\bar{b}$ to be revtjces of $a^{\star}$ dual 2 -cells that have $a$ and $b$ (respectively) on their boundaries, and then forlow a path in the 1-skeleton of the union of 2-cells dual to the vertices on a geodesic in $G^{\star}$ from $\bar{a}$ to $\bar{b}$. So $\operatorname{Diam}(G) \leqslant$ $\lambda\left(\operatorname{Diam}\left(G^{\star}\right)+1\right)$. Thus Conjecture 2.1 implies Conjecture 2.2 .

All the other implications are immediate from the definitions.

It is not hard to verify that Conjectures $2.1-2.4$ hold when $\lambda<3$. It would seem that the full intricacy of these conjectures is contained in the case $\lambda=3$. Establishing Conjecture 2.4 for $\lambda=3$ is sufficient for the group theoretic applications in this article on account of the well-known technique of triangulating a finite presentation $\mathcal{P}=\langle\mathcal{A} \mid \mathcal{R}\rangle$ of a group $\Gamma$ : obtain a new finite presentation for $\Gamma$ in which every relator has length at most three by a finite sequence of the following operations. If some $r \in \mathcal{R}$ is a concatenation $w_{1} w_{2}$ of two words $w_{1}$ and $w_{2}$ both of length at least 2, then add a new generator $a$ to $\mathcal{A}$, and in $\mathcal{R}$ replace $r$ by the two words $a^{-1} w_{1}$ and $a w_{2}$.

\section{Examples}

Figure 1 shows the fourth of a family $G_{n}$ of planar graphs that have $2^{n}+1$ vertices. The vertex valences of the dual graphs $G_{n}{ }^{\star}$ are at most three. So $G_{n}$ falls under the scope of Conjectures 2.1 and 2.2. In Figure $1 a$ we show $T^{\star}$, a maximal geodesic tree in $G_{n}{ }^{\star}$ based at the vertex at infinity (drawn with dotted lines). The diameter of $T^{\star}$ is $2 n$. The complementary maximal tree $T$ in $G_{n}$ (drawn with heavy lines) has diameter $2^{n}$. Figure $1 b$ 

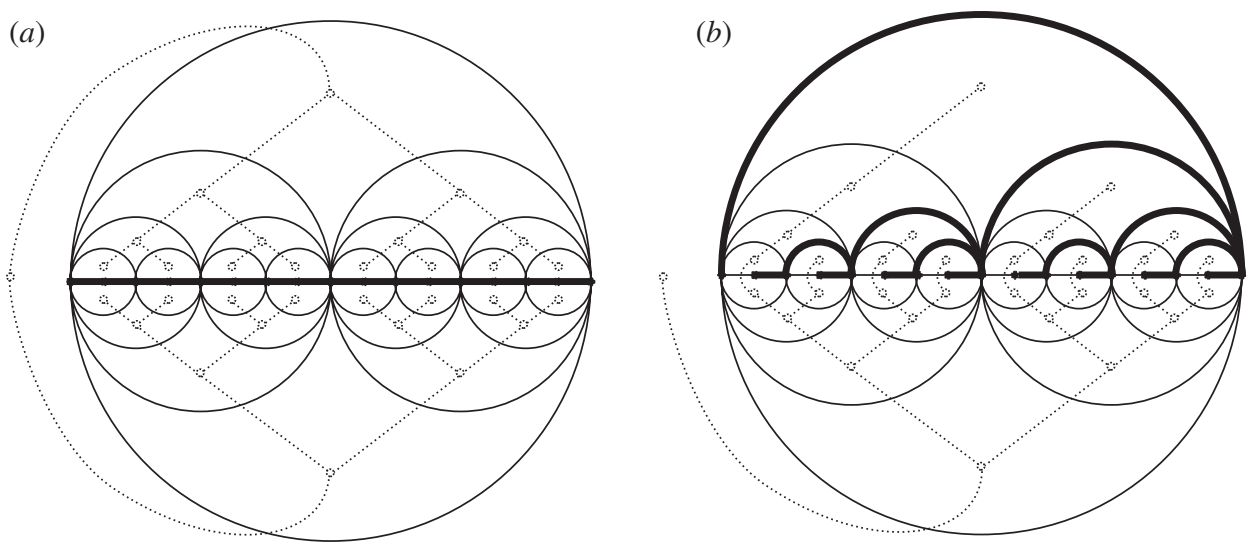

Figure 1. Two complementary pairs of maximal trees for a planar graph $G_{4}$.

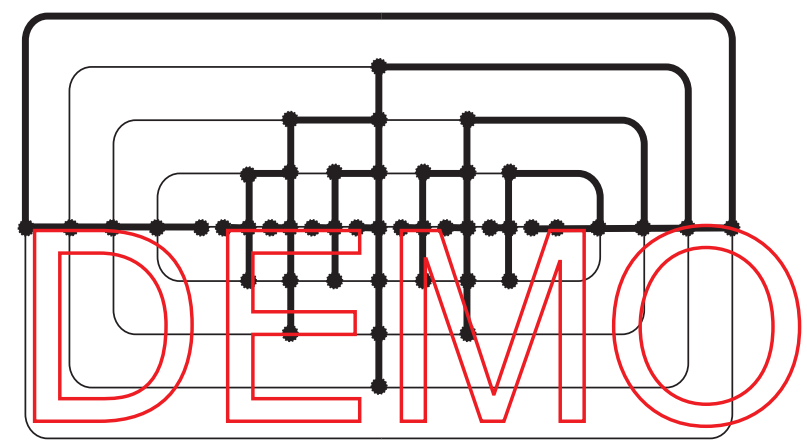

Figure 2. A maximal tree in the diagram $\tilde{G}_{4}$.

shows a different complementary pair of maximal trees $\left(T, T^{\star}\right)$. Here $\operatorname{Diam}(T)=2 n-1$ and $\operatorname{Diam}\left(T^{\star}\right)=2 n$. Thus this family of examples shows that in order to satisfy the demands of Conjectures 2.1 and 2.2, it does not suffice to take the maximal trees in the dual graphs to be geodesic trees.

The fourth graph of a related family $\tilde{G}_{n}$ is shown in Figure 2. In $\tilde{G}_{n}$ each vertex has valence at most 5 and in the dual $\tilde{G}_{n}^{\star}$ the vertex valences are at most 4 . So this family (and their duals) are within the scope of Conjecture 2.3. Like the case of the graphs $G_{n}$, taking $T^{\star}$ to be a maximal geodesic tree in $\tilde{G}_{n}^{\star}$ based at the vertex at infinity does not produce a pair of complementary maximal trees that satisfy the requirements of Conjecture 2.3. However, taking $T$ to be the maximal tree shown with heavy lines, we get a complementary pair with $\operatorname{Diam}(T) \sim \operatorname{Diam}\left(T^{\star}\right) \sim n$.

An example from a family within the scope of Conjecture 2.4 is shown in Figure 3 in $\S 5$. These graphs are 1-skeleta of van Kampen diagrams (defined in $\S 5$ ) for the words $\left[b^{n}, a^{-1}\right]\left[b^{n}, a\right]$ over $\left\langle a, b \mid b^{-1} a b=a^{2}\right\rangle$. All horizontal edges are labelled by $a$ and all vertical edges by $b$. Figure $3 a$ shows a choice of maximal trees that gives complementary pairs that fail the conjecture. Figure $3 b$ shows a maximal tree $T$ such that $\operatorname{Diam}(T) \sim$ $\operatorname{Diam}\left(T^{\star}\right) \sim n$. 

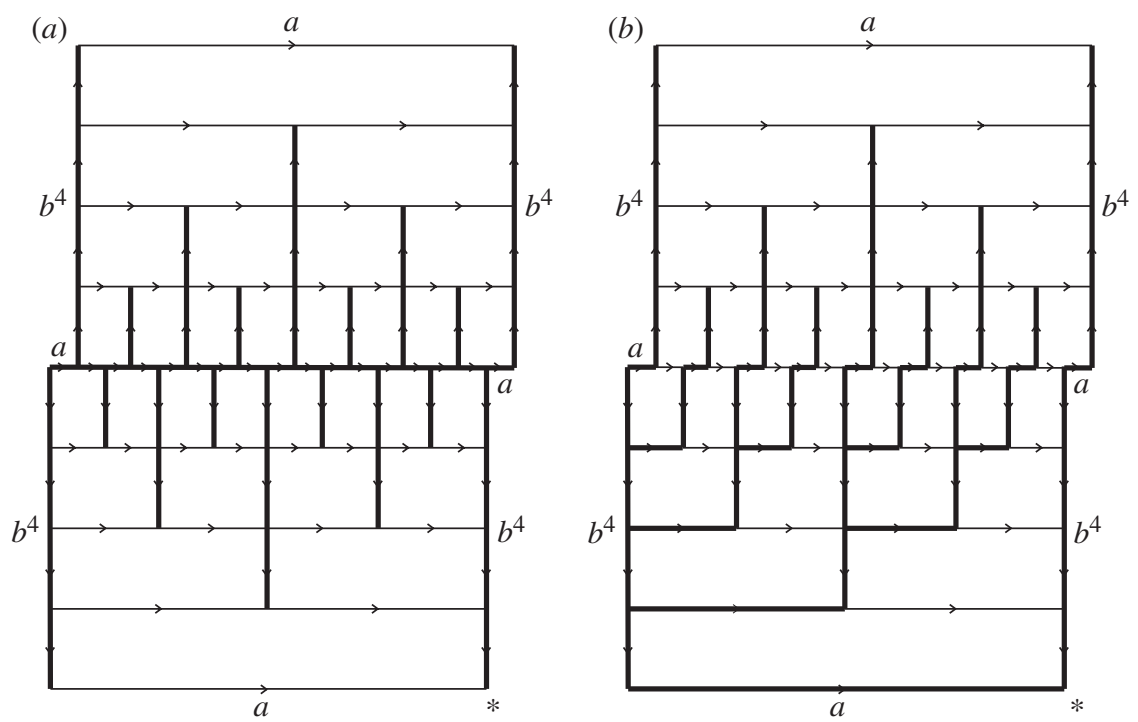

Figure 3. Maximal trees in the 1-skeleton of a van Kampen diagram $\hat{D}_{4}$.

\section{Singular disc diagrams}

Definition 4.1. A singutar dis diagram $D=S \times / 4$ a combipatorial 2-complex that can be obtained from some finite-erhbinatorial 2-con plex $S$ homeomorphic to the 2-sphere by removin $\$$ the interior of a 2 -cell

So $D$ is a finite, planar, eontractible, corhbirlatorial 2 complex; in general, $D$ need not be a topological 2-disc but rather is a tree-like arrangement of topological discs connected by one-dimensional arcs. For brevity, we refer to $D$ as a diagram.

A finite, connected, undirected graph* $G$ embedded in the 2 -sphere induces a combinatorial 2-complex structure $S$ with 1-skeleton $G$. Associated with $S$ is the dual 2-complex $S^{\star}$ that has a face dual to each vertex of $S$, an edge dual to each edge of $S$, and a vertex dual to each face of $S$. The 1-skeleton of $S^{\star}$ is $G^{\star}$, the dual graph of $G$.

Definition 4.2 (diagram measurements). A diagram measurement $\mathrm{M}$ assigns a real number $\mathrm{M}(D)$ to a diagram $D$. We will be concerned with the following diagram measurements.

We define a number of measurements that capture aspects of the geometry of a diagram $D$ with a base vertex $v_{0}$ in $\partial D^{(0)}$.

(i) The area Area $(D)$ is the number of 2-cells in $D$.

(ii) The diameter $\operatorname{Diam}(D):=\operatorname{Diam}_{v_{0}}(G)=\max \left\{d\left(v_{0}, a\right) \mid a \in G^{(0)}\right\}$, where $G:=D^{(1)}$.

(iii) The perimeter Perimeter $(D)$ is the length of the boundary circuit of $D$. This equals the valence of the vertex $e_{\infty}^{\star}$ at infinity in $G^{\star}$.

* Or 'multigraph' (see the footnote in $\S 1$ ). 
(iv) The gallery length $\mathrm{GL}(D)$ is the diameter of the dual graph $G^{\star}$, and so is essentially the dual concept to Diam.

(v) The measurement DGL $(D)$ is an upper bound for both diameter and gallery length. It is defined to be the minimum of $\operatorname{Diam}(T)+\operatorname{Diam}\left(T^{\star}\right)$ as $\left(T, T^{\star}\right)$ ranges over all complementary pairs of maximal trees for $G=D^{(1)}$.

(vi) $\operatorname{Dlog} \mathrm{A}(D)$ is defined to be $\operatorname{Diam}(D)+\log _{2}(\operatorname{Area}(D)+1)$.

(vii) The filling length $\mathrm{FL}(D)$ is the minimum filling length $\mathrm{FL}(\mathcal{S})$ amongst all shellings $\mathcal{S}$ of $D$.

The precise definition of a shelling as well as more details about filling length can be found in [10] or [12]. Roughly speaking, a shelling in a combinatorial null-homotopy of $D$ down to the base vertex $v_{0}$.

It is a simple consequence of the definitions that Conjecture 2.4 can be reformulated in terms of the diagram measurements $\mathrm{GL}(D)$ and $\mathrm{DGL}(D)$.

Conjecture 4.3. Fix $\lambda>0$. There exists $K>0$ such that if $D$ is a diagram in which every face has boundary circuit of length at most $\lambda$, then

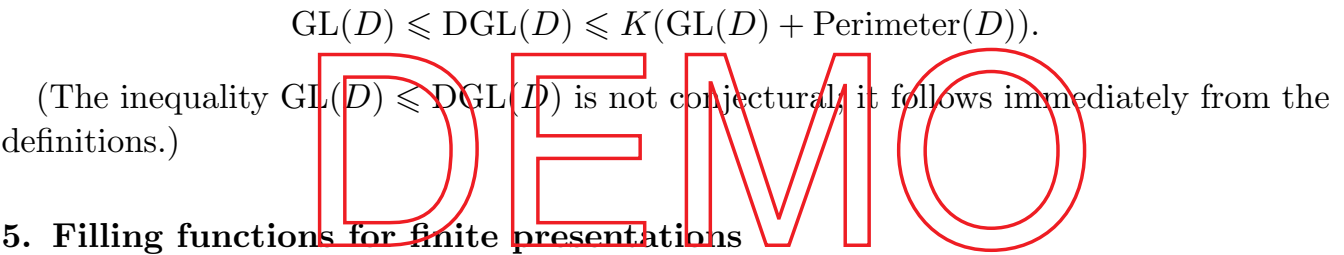

Here we present some of the notions of geometric group theory that we need before we can explain the applications of the conjectures.

Filling functions for a finite presentation $\mathcal{P}=\langle\mathcal{A} \mid \mathcal{R}\rangle$ of a group $\Gamma$ are defined using measurements of diagrams $D_{w}$ associated with a proof that a word* $w$ represents 1 in $\Gamma$. So a filling function captures an aspect of the geometry of the word problem for $\mathcal{P}$. The diagrams in question are called van Kampen diagrams and we recall their definition below.

Denote the length of $w$ by $\ell(w)$. Let $\mathcal{K}^{2}$ be the compact 2-complex with fundamental group $\Gamma$ associated with $\mathcal{P}$ : to construct $\mathcal{K}^{2}$ take a wedge of $|\mathcal{A}|$ circles, label each circle by an element of $\mathcal{A}$ and orient them, and then attach a $\ell(r)$-sided 2-cell for each $r \in \mathcal{R}$ with $r$ describing its attaching map. Let $C(\mathcal{P})=\widetilde{\mathcal{K}^{2}}$ denote the Cayley 2 -complex associated with $\mathcal{P}$. The Cayley graph of $\mathcal{P}$ is the 1 -skeleton of $C(\mathcal{P})$, and the 0 -skeleton is identified with $\Gamma$ so that the combinatorial metric on $C(\mathcal{P})$ agrees with the word metric $d_{\mathcal{P}}$ on $\Gamma$. Each edge of the Cayley graph inherits an orientation from $\mathcal{K}^{2}$ as well as a label by an element of $\mathcal{A}$.

A word $w$ in $\Gamma$ such that $w=1$ in $\Gamma$ is said to be null-homotopic, or is referred to as an edge-circuit, because it defines a loop in $C(\mathcal{P})^{(1)}$ based at 1 (say).

* Words are strings on letters in $\mathcal{A}$ and their formal inverses, that is, words are the elements of the free monoid $\left(\mathcal{A} \cup \mathcal{A}^{-1}\right)^{\star}$. 
Definition 5.1. Suppose $w$ is a null-homotopic word. Then a diagram $D_{w}=S \backslash e_{\infty}$ with base vertex $v_{0}$ is a $\mathcal{P}$-van Kampen diagram* for $w$ when there is a combinatorial $\dagger$ map $\Phi:\left(D_{w}, v_{0}\right) \rightarrow(C(\mathcal{P}), 1)$ such that $\left.\Phi\right|_{\partial D_{w}}$ is the edge-circuit $w$.

Each edge of $D_{w}$ inherits a direction from its image in $C(\mathcal{P})^{(1)}$ and a labelling by an element of $\mathcal{A}$. So the word one reads around the boundary of each of the 2-cells of $D_{w}$ is a cyclic conjugate of an element of $\mathcal{R} \cup \mathcal{R}^{-1}$, and starting at the base vertex $v_{0}$ one reads $w$ (by convention anticlockwise) around $\partial D$.

For an edge-circuit $w$ define

$$
\mathrm{M}(w):=\min \left\{\mathrm{M}\left(D_{w}\right) \mid D_{w} \text { is a van Kampen diagram for } w\right\},
$$

where $\mathrm{M}$ is a diagram measurement (see Definition 4.2 ). We write $\mathrm{M}_{\mathcal{P}}(w)$ when we wish to stress the finite presentation concerned.

We mention some equivalent definitions of $\operatorname{Area}(w), \operatorname{Diam}(w)$ and $\operatorname{FL}(w)$. It is a consequence of van Kampen's lemma (see $[\mathbf{5 , 1 7}]$ or $[\mathbf{1 8}]$ ) that $\operatorname{Area}(w)$ is the least $N$ such that there is an equality

$$
w=\prod_{i=1}^{N} u_{i}^{-1} r_{i} u_{i}
$$

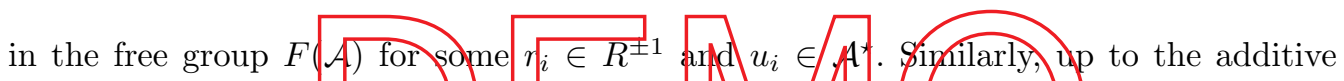
constant $\max \{\ell(r) \mid r \in R\}$, the dianmeter-Piand (2u) is the min imal bound on the length of the conjugating elements $u_{i}$ in equalities (5.1) Proposition 1 in [10] says/that $\mathrm{FL}(w)$ is the minimal bound on thelength of words one encounters in the prosess of applying defining relators $\ddagger$ to reduce $w$ to the empty word. There is another interpretation of Area $(w)$ in this context: it is the number of times relators are applied in the course if the reduction. These formulations of the definitions of $\operatorname{Area}(w)$ and $\mathrm{FL}(w)$ are particularly significant because they reveal the resulting filling functions Area $: \mathbb{N} \rightarrow \mathbb{N}$ and FL $: \mathbb{N} \rightarrow \mathbb{N}$, defined below, to be the non-deterministic complexity measures of the crude method of attacking the word problem in $\mathcal{P}$ by exhaustively applying relators.

Now we come to the definition of the filling functions.

Definition 5.2 (filling functions). For a diagram measurement $M$ we define a filling function $\mathrm{M}: \mathbb{N} \rightarrow \mathbb{N}$ for $\mathcal{P}$ by

$$
\mathrm{M}(n):=\max \{\mathrm{M}(w) \mid \text { edge-circuits } w \text { with } \ell(w) \leqslant n\} .
$$

In particular,

(i) Area $: \mathbb{N} \rightarrow \mathbb{N}$ is known as the Dehn function,

(ii) Diam : $\mathbb{N} \rightarrow \mathbb{N}$ is the minimal isodiametric function,

* We omit the $\mathcal{P}$ when there is no potential ambiguity.

$\dagger$ A combinatorial map sends $n$-cells homeomorphically onto $n$-cells for all $n$.

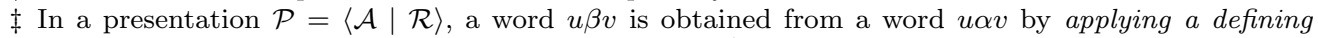
relator when a cyclic conjugate of the word $\alpha^{-1} \beta$ is in $\mathcal{R}^{ \pm 1}$. 
(iii) GL : $\mathbb{N} \rightarrow \mathbb{N}$ is the gallery length function, and

(iv) FL: $\mathbb{N} \rightarrow \mathbb{N}$ is the filling length function.

An isoperimetric (respectively, isodiametric) inequality for $\mathcal{P}$ is provided by any function $f: \mathbb{N} \rightarrow \mathbb{N}$ such that Area $(n) \leqslant f(n)$ (respectively, $\operatorname{Diam}(n) \leqslant f(n)$ ) for all $n$.

There are many references to isoperimetric functions, Dehn functions and isodiametric functions in the literature; $[\mathbf{5}]$ and $[\mathbf{8}]$ are surveys. The filling length function is discussed extensively in [10] and has an important application in [13]. We introduced GL and DGL in [12], and DlogA, which will play an important role in $\S 7$, is new.

The word problem for $\mathcal{P}$ is solvable if and only if any one (and hence all-see $\S 9$ ) of the filling functions Area, GL, DGL, DlogA and FL is bounded above by a recursive function (see $[8]$ ).

The following equivalence relation on functions $\mathbb{N} \rightarrow \mathbb{N}$ is well known.

Definition 5.3 ( $\simeq$-equivalence). For two functions $f, g: \mathbb{N} \rightarrow \mathbb{N}$ we say that $f \preceq g$ when there exists $C>0$ such that $f(n) \leqslant C g(C n+C)+C n+C$ for all $n$, and we say $f \simeq g$ if and only if $f \preceq g$ and $g \preceq f$.

For example, for $p, q \geqslant 1$ we have $n^{p} \simeq n^{q}$ if and only if $p=q$.

Recall that Conjecture 2.4 about planar graphs was reformulated in terms of diagram measurements GL and DGL in Conjecture 4.3. If Conjecture 4.3 is true, then so is the following.

Conjecture 5.4. The fillins tunctions GL and $D$ GL for any given finite group presentation $\mathcal{P}$ are $\simeq$-equivalent.

It is important to nete that the filling furctions are defined for specific finite presentations for groups. However, up to $\simeq$-equivalence, Area and FL depend only on the group. Indeed both are quasi-isometry invariants up to $\simeq$-equivalence (see [1] and Theorem 8.1 of this article). The situation for GL is a little more complicated. One needs the following notion of fattening a presentation $\mathcal{P}=\langle\mathcal{A} \mid \mathcal{R}\rangle$ by adding to $\mathcal{A}$ an extra generator $z$ which represents 1 in the group, and adding a number of extra relations involving $z$ to $\mathcal{R}$.

Definition 5.5 (fat presentations). One obtains a fat presentation $\mathcal{P}^{\prime}$ from a presentation $\mathcal{P}=\langle\mathcal{A} \mid \mathcal{R}\rangle$ by adjoining an extra generator $z$ to $\mathcal{A}$ as follows:

$$
\mathcal{P}^{\prime}:=\left\langle\mathcal{A} \cup\{z\} \mid \mathcal{R} \cup\left\{z, z^{2}, z z^{-1}, z^{3}, z^{2} z^{-1}\right\} \cup\{[a, z]: a \in \mathcal{A} \cup\{z\}\}\right\rangle .
$$

It is proved in [12] that if $\mathcal{P}$ and $\mathcal{Q}$ are two finite presentations for the same group $\Gamma$, then the gallery length functions $\mathrm{GL}_{\mathcal{P}^{\prime}}$ and $\mathrm{GL}_{\mathcal{Q}^{\prime}}$ of the fat presentations $\mathcal{P}^{\prime}$ and $\mathcal{Q}^{\prime}$ are $\simeq$-equivalent. This result is shown in Theorem 8.1 of this article to continue to hold under the weaker hypothesis that $\mathcal{P}$ and $\mathcal{Q}$ present quasi-isometric groups.

In our study of central extensions, we will need to monitor not only how the gallery length functions of two finite presentations of the same groups are related, but also how simultaneous area and gallery length bounds change. The following definition gives us the appropriate notation and the subsequent proposition, which is [12, Scholium 4.7], gives us the control we will require. 
Definition 5.6. We say that a pair $(f, g)$ of functions $f, g: \mathbb{N} \rightarrow \mathbb{N}$ is an (Area, GL)pair for the finite presentation $\mathcal{P}$ when, for every edge-circuit $w$ in the Cayley graph of $\mathcal{P}$, there exists a van Kampen diagram $D_{w}$ with $\operatorname{Area}\left(D_{w}\right) \leqslant f(\ell(w))$ and $\operatorname{GL}\left(D_{w}\right) \leqslant$ $g(\ell(w))$.

Proposition 5.7. Suppose that $\mathcal{P}$ and $\mathcal{Q}$ are two finite presentations for the same group $\Gamma$, and that $\mathcal{P}^{\prime}$ and $\mathcal{Q}^{\prime}$ are their fattenings. If $\left(f_{\mathcal{P}^{\prime}}, g_{\mathcal{P}^{\prime}}\right)$ is an (Area, GL)-pair for $\mathcal{P}^{\prime}$, then there is an (Area, GL)-pair $\left(f_{\mathcal{Q}^{\prime}}, g_{\mathcal{Q}^{\prime}}\right)$ for $\mathcal{Q}^{\prime}$ such that $f_{\mathcal{P}^{\prime}} \simeq f_{\mathcal{Q}^{\prime}}$ and $g_{\mathcal{P}^{\prime}} \simeq g_{\mathcal{Q}^{\prime}}$.

\section{The geometry of central extensions}

We begin an examination of the geometry of central extensions $\hat{\Gamma}$ of finitely presentable groups $\Gamma$ with the case where the central abelian kernel is $\mathbb{Z}$ :

$$
1 \rightarrow \mathbb{Z} \rightarrow \hat{\Gamma} \rightarrow \Gamma \rightarrow 1
$$

If $\mathcal{P}=\langle\mathcal{A} \mid \mathcal{R}\rangle$ is a presentation for $\Gamma$, then there is a presentation for $\hat{\Gamma}$ of the form

$$
\left.\hat{\mathcal{P}}=\langle\hat{\mathcal{A}} \mid \hat{\mathcal{R}}\rangle=\langle\mathcal{A} \cup\{z\}| r=z^{n_{r}} \text { for } r \in \mathcal{R} ;[z, a] \text { for all } a \in \mathcal{A}\right\rangle .
$$

Define $M:=\max \left\{\left|n_{r}\right| \mid r \in \mathcal{R}\right\}$.

Proposition 6.1 (infinite cyclic kerne1 dase)/supp se $w$ is a word in $\left(\mathcal{A}^{ \pm 1}\right)^{\star}$ that represents 1 in $I$, and oo represents $z^{n}$ in $\hat{\Gamma}$ for spnee integer $m$. Assume $D$ is a $\mathcal{P}$-van Kampen diagram for an edge-circuit w. Let f be a nadximal tree in $G=D^{(1)}$. As
usual, the base vertex of $D$ is called w.

There is a $\hat{\mathcal{P}}$-van Kampen diagram $\hat{D}$ for $w z^{-m}$ such that

$$
\begin{aligned}
\operatorname{Diam}\left(\hat{G}^{\star}\right) & \leqslant 2 \operatorname{Diam}_{v_{0}}(T)+\operatorname{Diam}\left(T^{\star}\right), \\
\operatorname{Area}(\hat{D}) & \leqslant M \operatorname{Diam}_{v_{0}}(T) \operatorname{Area}(D)+\operatorname{Area}(D),
\end{aligned}
$$

where $\hat{G}^{\star}$ is the graph dual to $\hat{G}=\hat{D}^{(1)}$.

Proof. We will inflate $D$ to produce a $\hat{\mathcal{P}}$-van Kampen diagram $\hat{D}$ for $w z^{-m}$. The defining relators $r$ in $\mathcal{R}$ are expanded to relators $r z^{-n_{r}}$ in $\hat{\mathcal{R}}$ and the resulting introduction of the $z$-edges in the boundaries of the corresponding 2-cells prevent them fitting together in the same arrangement as in $D$ to make a $\hat{\mathcal{P}}$-van Kampen diagram. The remedy is to use the electrostatic model as explained in the following three steps and illustrated in Figures 4 and 5.

1. Charge the diagram $D$. Fix an embedding of $D$ in the plane. Each 2-cell $e$ in $D$ has its boundary labelled by some $r \in \mathcal{R}^{ \pm 1}$ read from a base vertex $v_{e} \in \partial e$. Inscribe a planar wedge of $\left|n_{r}\right| 2$-discs in $e$; each of these 2-discs has a boundary made up of one directed 1-cell whose initial and terminal vertices are both identified with $v_{e}$. Refer to each of these 2-discs as charges; each has a boundary loop labelled by $z$ and is directed in such a way that around the boundary loop of the (now altered) 2 -cell $e$ one reads the 


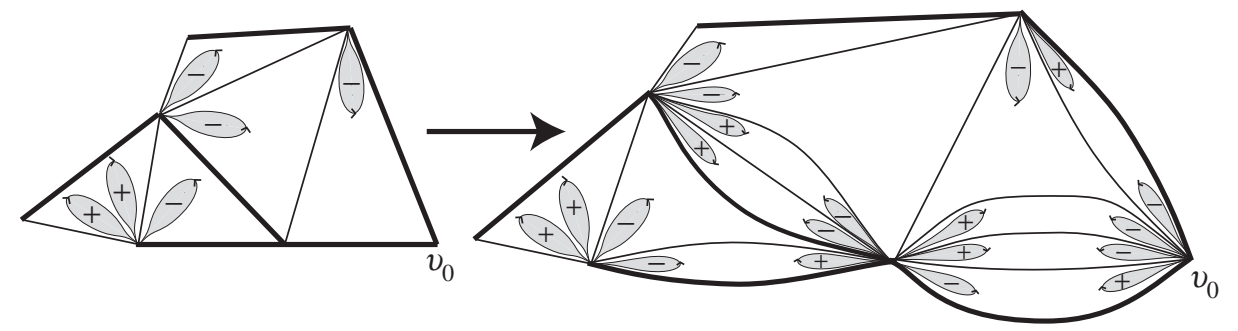

Figure 4. Discharge of excess charge along a maximal tree $T$ in a van Kampen diagram to $v_{0}$.

relator $\left(r z^{-n_{r}}\right)^{ \pm 1}$. We say that a charge is positive or negative depending on whether one reads $z$ in a clockwise or anticlockwise direction. Call the charged diagram $D^{\mathrm{c}}$.

Figure $6 a$ is an example of a charged diagram. Here charges are inserted to inflate the relators $[x, y]$ to $[x, y] z^{-1}$.

2. Discharge along the maximal tree $\boldsymbol{T}$. To get a van Kampen diagram for $w$ from $D^{\mathrm{c}}$, a natural approach is to blow up each vertex and then to fold together the edges labelled $z$. However, for this to work it is necessary that the charge at each vertex $v$ is electrostatically neutral, that is, there are the same number of positive as negative charges attached to $v$. The way we achieve this is to discharge the diagram along $T$. At each vertex pair off the positive charges with the freatife charges and refer to the remaining unpaired charges as the excess charges. We now discharge the excess charge to $v_{0}$ as follows. We transfer all the excess charge at each vertex $v$ in $D^{c}$ along a path $p$ in $T$ to $v_{0}$ using a string of digons. Each 1-cell in $p$ is doubled te sreate digon (one of the two resulting edges is chosen to be in the maximal tree of the new diagram), and then a positive charge is added at one end of the digon and a negative charge at the other. The charged digons are aligned along $p$ in such a way that at every vertex on $p$ apart form $v$ and $v_{0}$, one positive and one negative charge is added. The effect is then that the net charge at $v$ is altered by one at the expense of the charge at $v_{0}$. The net charge at each of the other vertices is left unchanged.

3. Blow-up and fold. From a diagram where all vertices apart from $v_{0}$ are electrically neutral, we produce a diagram over the presentation $\hat{\mathcal{P}}$, that is, a diagram in which all the 2-cells have boundaries labelled by words in $\hat{\mathcal{R}}^{ \pm 1}$. The wedge of 2-disc charges at each vertex $v \neq v_{0}$ is blown up into a 2-cell with boundary word made up of $z$ and $z^{-1}$ s. The exponent sum of the $z^{ \pm 1}$ s comprising this word is zero as the net charge at $v$ is neutral. Adjacent $z z^{-1}$ or $z^{-1} z$ pairs are then folded together until the blown-up 2-cell is entirely eliminated. (This process of folding does not need to be specified uniquely.)

The blow-up procedure at $v_{0}$ differs in that we cut out the charges at $v_{0}$ so as to introduce a subword $u$ made up of $z$ and $z^{-1}$ s into the diagram's boundary word. So the boundary word of the new diagram is $u w$. But then $u=z^{-m}$ in $\hat{\Gamma}$ because $w=z^{m}$ in $\hat{\Gamma}$. Thus the exponent sum of the letters $z$ comprising $u$ is $-m$ and folding together adjacent $z z^{-1}$ or $z^{-1} z$ pairs in $u$ gives a diagram $\hat{D}$ with boundary circuit $z^{-m^{\prime}} w$ for some $m^{\prime}$. And because $w=z^{m^{\prime}}=z^{m}$ we have $m=m^{\prime}$. 


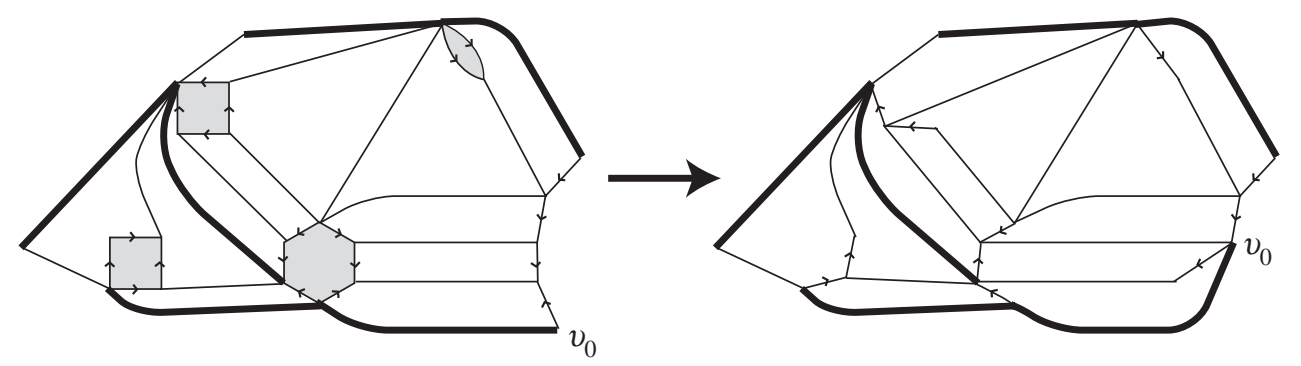

Figure 5. The effect of blowing up and then folding the diagram from Figure 4.

(6)

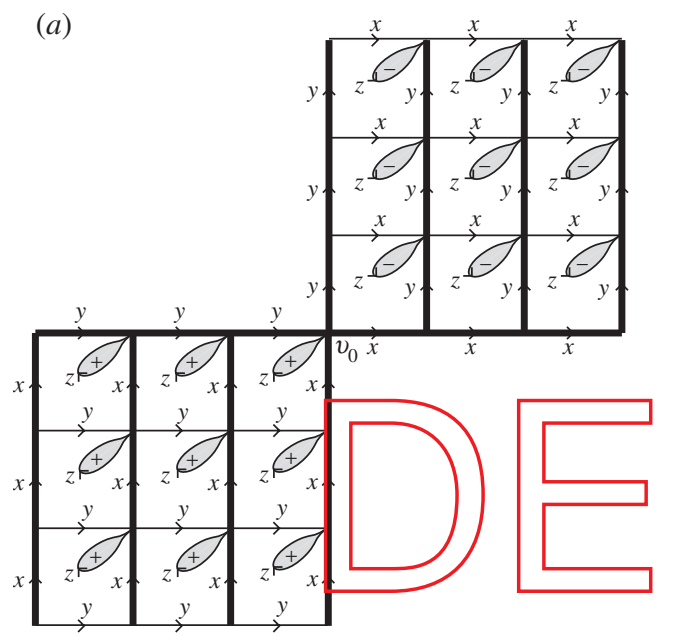

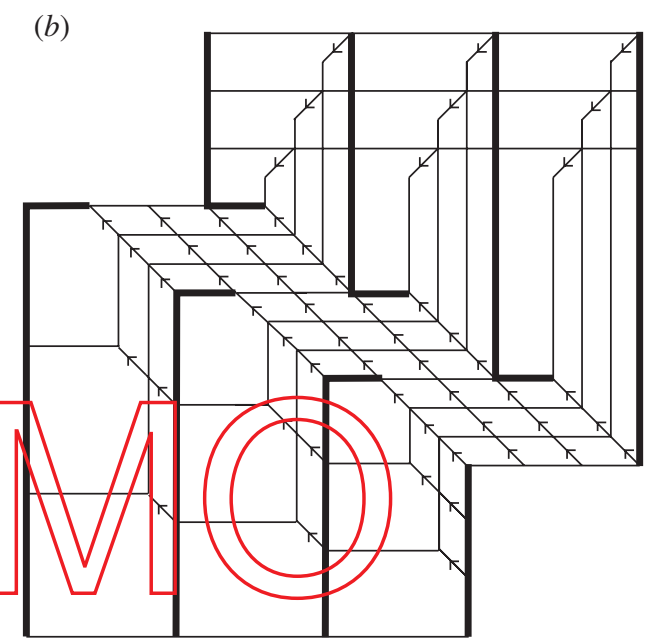

Figure 6. A van Kampen diagram for the word $\left[x^{-3}, y^{-3}\right]\left[y^{3}, x^{3}\right]$ over $\langle x, y \mid[x, y]\rangle$ charged and then inflated to a diagram over the three-dimensional integral Heisenberg group $\langle x, y, z|[x, y]=$ $z,[x, z]=1,[y, z]=1\rangle$. In $(b)$ the edges whose directions have been marked are the $z$-edges.

The three-dimensional integral Heisenberg group $\langle x, y, z|[x, y]=z,[x, z]=1,[y, z]=$ $1\rangle$ is an example of a central extension with kernel $\mathbb{Z}=\langle z\rangle$. The effect of charging a diagram for the word $\left[x^{-3}, y^{-3}\right]\left[y^{3}, x^{3}\right]$ over $\langle x, y \mid[x, y]\rangle$, discharging along a maximal tree $T$ (depicted using heavier lines), blowing up and then folding is illustrated in Figure 6. In this case each relator $[x, y]$ is inflated to include one $z$-edge and the discharge procedure collects nine positive and nine negative charges at $v_{0}$. Blowing up at $v_{0}$ inserts a subword $z^{9} z^{-9}$ into the boundary word, which is then folded into the interior of the final diagram.

We now prove the bounds (6.1) and (6.2) for $\hat{D}$. A copy of the maximal tree $T^{\star}$ in $G^{\star}$ can be found in $\hat{G}^{\star}$, and this can be reached from any vertex in $G^{\star}$ by following a path along one of the $z$-corridors. These $z$-corridors have length at most $\operatorname{Diam}_{v_{0}}(T)$, hence the bound (6.1). For the area bound (6.2), let $Q\left(D^{\mathrm{c}}\right)$ denote the total charge $\sum_{e}\left|n_{r_{e}}\right|$, summed over the Area $(D)$ 2-cells $e$ of the $D$, where $r_{e}$ is the relator that is the boundary word for $e$. Then $Q(\bar{D}) \leqslant M$ Area $(D)$ is an upper bound for the total number of $z$-corridors introduced into $D$ when making $\hat{D}$. Each of these corridors is made up of 
at most $\operatorname{Diam}_{v_{0}}(T)$ 2-cells. So the first term on the right-hand side of (6.2) is an upper bound on the total contributions made by the $z$-corridors to the area of $\hat{D}$. The remainder of $\hat{D}$ is the inflated Area $(D)$ 2-cells from $D$, and thus we deduce (6.2).

The methods above can be adapted to the case where the central abelian kernel is a finite cyclic group $C_{q}$ :

$$
1 \rightarrow C_{q} \rightarrow \hat{\Gamma} \rightarrow \Gamma \rightarrow 1
$$

If $\mathcal{P}=\langle\mathcal{A} \mid \mathcal{R}\rangle$ is a presentation for $\Gamma$, then there is a presentation for $\hat{\Gamma}$ of the form

$$
\left.\hat{\mathcal{P}}=\langle\hat{\mathcal{A}} \mid \hat{\mathcal{R}}\rangle=\langle\mathcal{A} \cup\{z\}| r=z^{n_{r}} \text { for } r \in \mathcal{R} ;[z, a] \text { for all } a \in \mathcal{A} ; z^{q}\right\rangle .
$$

We can assume that $0 \leqslant n_{r}<q$ for all $r \in \mathcal{R}$. The conclusion of the following proposition differs from that of Proposition 6.1 in the bound on the area of $\hat{D}$.

Proposition 6.2 (finite cyclic kernel case). Suppose $w$ is a word in $\left(\mathcal{A}^{ \pm 1}\right)^{\star}$ that represents 1 in $\Gamma$, and so represents $z^{m}$ in $\hat{\Gamma}$ for some non-negative integer $m<q$. Assume that $D$ is a $\mathcal{P}$-van Kampen diagram for an edge-circuit $w$. Let $T$ be a maximal tree in $G=D^{(1)}$. Let $C:=\max \{\ell(r) \mid r \in \mathcal{R}\}$.

There is a $\hat{\mathcal{P}}$-van Kampen diagram $\hat{D}$ for $w z^{-m}$ such that

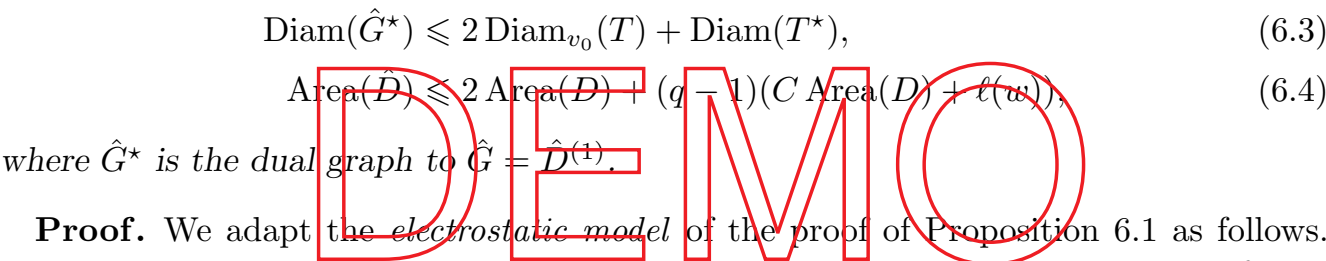
We charge the diagram (this time all the charges are positive because $0 \leqslant n_{r}<q$ for all $r \in \mathcal{R}$ ) and then discharge to $v_{0}$ along the maximal tree $T$ as before. But then before blowing up and folding the diagram we remove digons as follows. Let $v$, if it exists, be an edge a maximal distance from $v_{0}$ in $T$ such that there are at least $q$ digons along the first edge $e$ on the geodesic $\gamma$ in $T$ from $v$ to $v_{0}$. Then there are at least $q$ digons along every edge of $\gamma$. We remove $q$ digons from each edge of $\gamma$. Perform this removal of digons repeatedly until no $v$ can be found. In this way arrive at a diagram in which the number of digons along each edge is no more than $q-1$, and the net charge at each vertex is $0 \bmod q$. We then produce the diagram $\hat{D}$ by blowing up and folding as before, except that this time if a vertex $v \neq v_{0}$ has total charge $k q$, then $k$ faces labelled $z^{-q}$ will be inserted into the diagram. A word $z^{l}$ will be inserted into the boundary of the diagram at $v_{0}$ with $l=m \bmod q$, and we reduce this to $z^{m}$ by attaching $k$ faces labelled $z^{-q}$, where $l=k q+m$. The result is a diagram $\hat{D}$ for $w z^{-1}$.

The inequality (6.3) on $\operatorname{Diam}\left(\hat{G}^{\star}\right)$ is proved in the same way as $(6.1)$. The area bound (6.4) arises from considering the following three contributions to faces in $\hat{D}$ : the Area $(D)$ faces in $D$ are inflated to faces in $\hat{D}$; each edge in $T$, of which there are at most $C$ Area $(D)+\ell(w)$, is fattened to no more than $(q-1)$ digons before the blowing-up and folding procedure, and each of these digons gives a face in $\hat{D}$ with boundary label $[a, z]^{ \pm 1}$ for some $a \in \mathcal{A}$; and the number of $z^{-q}$ faces in $\hat{D}$ is at most $\operatorname{Area}(D)$ because at most $(q-1)$ Area $(D) z$-edges are introduced when $D$ is charged. 
The propositions above allow us to formulate results that give restrictions on how the geometry of the word problem of a group can change when one moves to a central extension. These results are framed in terms of '(Area, GL)-pairs' (see Definition 5.6) and the related notion of '(Area, DGL)-pairs' which are identically defined except with the filling function DGL (defined in $\S 5$ ) replacing GL.

Theorem 6.3. Suppose that $1 \rightarrow \Lambda \rightarrow \hat{\Gamma} \rightarrow \Gamma \rightarrow 1$ is a central extension of the finitely presented group $\Gamma$, and that $(f, g)$ is an (Area, DGL)-pair for a finite presentation $\mathcal{P}$ of $\Gamma$.

(i) If $\Lambda=\mathbb{Z}$, then, up to a common multiplicative constant, $\left(f(n) g(n)+n^{2}, g(n)+n\right)$ is an (Area, GL)-pair for the finite presentation $\hat{P}$ of Proposition 6.1 for $\hat{\Gamma}$.

(ii) If $\Lambda=C_{q}$, a finite cyclic group of order $q$, then, up to a common multiplicative constant, $(f(n)+n, g(n)+n)$ is an (Area, GL)-pair for the finite presentation $\hat{P}$ of Proposition 6.2 for $\hat{\Gamma}$.

Proof. Suppose $\hat{w}$ is a word in $\left(\hat{\mathcal{A}}^{ \pm 1}\right)^{\star}$ that represents 1 in the group $\hat{\Gamma}$ presented by $\hat{\mathcal{P}}=\langle\hat{\mathcal{A}} \mid \hat{\mathcal{R}}\rangle$. Let $n:=\ell(\hat{w})$ and let $w$ be the word obtained from $\hat{w}$ by removing all instances of the generator $z$ of the cyclic central kernel $\Lambda$. Then $w$ represents 1 in $\Gamma$ and $z^{m}$ in $\hat{\Gamma}$ for some integer $m$ (with $0 \leqslant m<q$ in the case of $\Lambda=C_{q}$ ). Let $D$ be a $\mathcal{P}$-van Kampen diagr an for $x$ exh ipiting the Area and/D\&L bomdsph the (Area, DGL)-

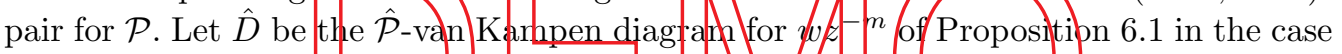
of $\Lambda=\mathbb{Z}$ and of Proposition 5. 2 in the case of $\Lambda=4$.

We attach a planar annulat diagram $A$ anound lehe poundary of D/to affect a transformation of $\hat{w}$ to $w z^{m}$ and thereby produce a van Kampen diagram for $\hat{w}$ as follows.

In the case $\Lambda=\mathbb{Z}$, the exponent sum of the occurrences of $z$ in $w$ equals $m$, and $A$ consists of $z$-corridors that collect the letters $z^{ \pm 1}$ in $\hat{w}$ together so that after cancelling $z z^{-1}$ pairs we have $w z^{-m}$. The length of each $z$-corridor is at most $n$ and so the increase in gallery length when $A$ is attached to $\hat{D}$ is at most $2 n$. The area of $A$ is at most $n^{2}$ since each letter $z$ is moved a distance at most $n$ using commutator relations.

When $\Lambda=C_{q}$, the exponent sum of the occurrences of $z$ in $w$ equals $m \bmod q$. We let $A$ be the annular diagram with outer boundary $\hat{w}$ and inner boundary $w z^{-m}$ that is obtained by moving letters $z$ around $\hat{w}$ using commutator relations as follows. We start at the left-hand end of the word $w$ and read along the word from left to right. Each time we come to a letter $z^{ \pm 1}$ we move it to the right until we either come to a $z^{\mp 1}$, in which case we cancel the two letters, or we come to a $z^{ \pm 1}$, in which case we carry this letter along also. As we progress through the word we pick up more and more letters $z^{ \pm 1}$ in this way, and if the number of letters we are carrying reaches $\pm q$, we apply the relator $z^{q}=1$ to cancel them all. When we reach the right-hand end of the word we have transformed the word to $w z^{-m}$ or $w z^{-m+q}$. In the latter case we use a relator $z^{q}=1$ to get the word $w z^{-m}$.

The total number of $z^{q}=1$ relations we use is at most $1+(n / q)$ and at any stage in the above process we are moving at most $q-1$ letters $z^{ \pm 1}$ through the word, so we use at most $(q-1) n$ commutator relations. Thus the area of $A$ is at most $n$, up to a 
multiplicative constant. It follows that attaching $A$ to $\hat{D}$ does not increase the gallery length by more than $n$, up to a multiplicative constant.

Recall that Conjecture 4.3 claims a close relationship between the diagram measurements GL and DGL.

Corollary 6.4. Assuming Conjecture 4.3 holds, Theorem 6.3 is true under the weaker hypothesis that $(f, g)$ is an (Area, GL)-pair.

Having considered the cases of a central extension with kernel $\mathbb{Z}$ or $C_{q}$, one is ready for the general case of a central extension with kernel an arbitrary finitely generated abelian group $\Lambda$.

Theorem 6.5. Assume Conjecture 4.3 holds. Let

$$
1 \rightarrow \Lambda \rightarrow \hat{\Gamma} \rightarrow \Gamma \rightarrow 1
$$

be a central extension of the finitely presented group $\Gamma$ with finitely generated abelian kernel $\Lambda$. Let $\mathcal{P}=\langle\mathcal{A} \mid \mathcal{R}\rangle$ be a finite presentation for $\Gamma$ and let $(f, g)$ be an (Area, GL)pair for $\mathcal{P}$. Then there is a finite presentation $\hat{\mathcal{P}}$ for $\hat{\Gamma}$ such that, up to a common multiplicative constant, $\left(f(n) g(n)+n^{2}, g(n)+n\right)$ is an (Area, GL)-pair for $\hat{\mathcal{P}}$.

Proof. We express $\Lambda_{\text {as a direct product }} \mathbb{P}^{k} \times C_{q_{1}} \times G_{q_{2}} \times \cdots C_{q_{j}}$ of cyclic groups

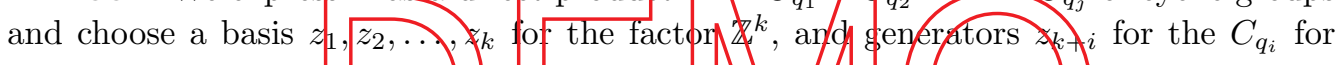
$i=1,2, \ldots, j$. $\hat{\mathcal{R}}$ is

Take the presentation $\hat{\mathcal{P}}$ for $\hat{\Gamma}$

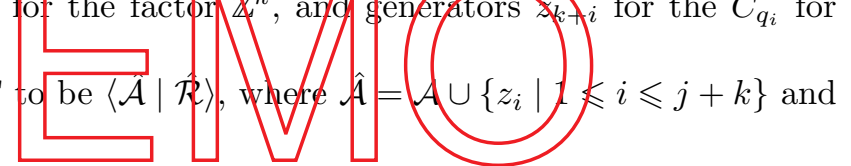

$$
\begin{aligned}
\left\{r=u_{r} \mid r \in \mathcal{R}\right\} & \cup\left\{\left[a, z_{i}\right] \mid a \in \mathcal{A}, 1 \leqslant i \leqslant j+k\right\} \\
& \cup\left\{\left[z_{i}, z_{i^{\prime}}\right] \mid 1 \leqslant i, i^{\prime} \leqslant j+k\right\} \\
& \cup\left\{z_{k+i}{ }^{q_{i}} \mid l \leqslant i \leqslant j\right\}
\end{aligned}
$$

with $u_{r}$ a word in the generators $z_{1}, z_{2}, \ldots, z_{k+j}$. Suppose $\hat{w}$ is a word in the generators of $\hat{\mathcal{P}}$ such that $\hat{w}=1$ in $\hat{\Gamma}$, and let $w$ be obtained from $\hat{w}$ by deleting all letters $z_{i}{ }^{ \pm 1}$ for all $i$. Then $w=1$ in $\Gamma$.

Choose a $\mathcal{P}$-van Kampen diagram $D$ for $w$ according to the (Area, GL)-pair for $\mathcal{P}$.

We construct intermediate central extensions by adding one central basis element $z_{i}$ at a time: that is, we pass from $\hat{\Gamma} /\left\langle z_{1}, z_{2}, \ldots, z_{i}\right\rangle$ to $\hat{\Gamma} /\left\langle z_{1}, z_{2}, \ldots, z_{i-1}\right\rangle$. On adding each $z_{i}$ we inflate the van Kampen diagram as per Corollary 6.4 to produce a van Kampen diagram for a word in which letters $z_{j}^{ \pm 1}$ are returned to their places in $\hat{w}$.

Inductively we see that the gallery length of the diagrams remains bounded by $g(\ell(\hat{w}))+n$ up to a multiplicative constant. However, the bound we get on the area of these diagrams from Corollary 6.4 increases by a factor of the gallery length with each successive central extension. But, looking back at (6.2) we see that the dominant term in the area estimate is the product term $\operatorname{Diam}_{v_{0}}(T) \operatorname{Area}(D)$ that comes from the contributions of the corridors that move central elements ('charges') along the tree $T$ when we blow up relators from $\mathcal{R}$. 
The total amount of charge moved throughout the $j+k$ successive central extensions is at most $M \operatorname{Area}(\bar{D})$, where $M$ is the maximum length of the words $u_{r}$. The diameter of the trees $T$ (and hence the length of the resulting corridors) remains bounded in terms of $g(\ell(\hat{w}))$. So the area of the final $\hat{\mathcal{P}}$-van Kampen diagram is controlled by $f(\ell(w)) g(\ell(w))$.

Recall that a group $\Gamma$ is nilpotent of class $c$ when the lower series is a central series, defined inductively by $\Gamma_{1}:=\Gamma$ and $\Gamma_{i+1}:=\left[\Gamma_{i}, \Gamma\right]$, terminates at $\Gamma_{c+1}=\{1\}$ :

$$
\Gamma=\Gamma_{1} \gtrless \Gamma_{2} \gtrless \cdots \geqslant \Gamma_{c+1}=\{1\} .
$$

For each $i$, the group $\Gamma_{i}$ is a central extension of $\Gamma_{i+1}$, and the presentation $\langle x \mid x\rangle$ of the trivial group admits the (Area, GL)-pair $(n, 2)$. So repeated application of Corollary 6.4 starting at the trivial group, together with Proposition 5.7, gives the following corollary.

Corollary 6.6. Assume Conjecture 4.3 holds. If $\mathcal{P}$ is a finite fat presentation for a finitely generated nilpotent group $\Gamma$ of class $c$, then, up to a common multiplicative constant, $\left(n^{c+1}, n\right)$ is an (Area, GL)-pair for $\mathcal{P}$.

The isoperimetric function $n^{c+1}$ (up to a multiplicative constant) would reproduce the result of $[\mathbf{1 3 - 1 5}]$. If Conjecture 43 holds, then by Theorem 1.3 the linear bound on the gallery length would also regapture the linea: pound or filling length of $[\mathbf{1 3}, \mathbf{2 0}]$.

\section{Controlling}
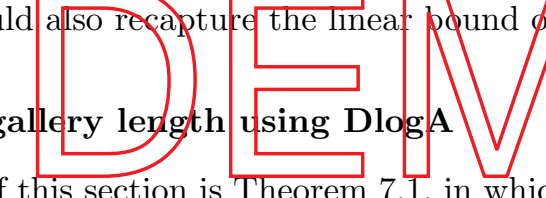

The main result of this section is Theorem 7.1, in which we give a convenient method of controlling the gallery length filling function. We prove that for a null-homotopic word $w$ in a fat finite presentation (see Definition 5.5), the sum of upper bounds for the diameter and the logarithm of the area, realizable simultaneously on one van Kampen diagram for $w$, can be used to estimate $\mathrm{GL}(w)$.

The theorem will be useful in $\S 8$, in which we give upper bounds on the gallery length of a number of different classes of groups. Also the results and constructions in this section contribute to the theory of how filling functions interrelate (see $\S 9$ ) and to understanding restricted filling functions (which we introduce in $\S 10$ ).

We use the filling function $\operatorname{Dlog} A: \mathbb{N} \rightarrow \mathbb{N}$ that arises from the diagram measurement:

$$
\operatorname{Dlog} \mathrm{A}(D):=\operatorname{Diam}(D)+\log _{2}(\operatorname{Area}(D)+1) .
$$

(See Definitions 4.2 and 5.2.)

Theorem 7.1. If $\mathcal{P}$ is a fat finite presentation, then its filling functions GL and DlogA satisfy $\mathrm{GL} \simeq \mathrm{D} \log \mathrm{A}$.

We set out the construction used in proving the bound GL $\preceq$ D logA in the following proposition. (Conclusion (iii) is not used in the proof of Theorem 7.1 but will be used to establish Proposition 7.5 and Theorem 10.3(c).) 
Proposition 7.2. Suppose $\mathcal{P}$ is a fat finite presentation for a group $\Gamma$.

There exists a constant $M>0$, depending only on $\mathcal{P}$, that satisfies the following. Suppose that $D$ is a $\mathcal{P}$-van Kampen diagram for an edge-circuit $w$ and that $T$ is a maximal tree in $D^{(1)}$. There is a $\mathcal{P}$-van Kampen diagram $\tilde{D}$ for $w$ in which every vertex has valence at most 12 and there is a maximal tree $\tilde{T}$ in $\tilde{D}^{(1)}$ such that

(i) $\operatorname{Area}(\tilde{D}) \leqslant M \operatorname{Area}(D)(1+\operatorname{Diam}(T))$,

(ii) $\operatorname{Diam}(\tilde{T}) \leqslant M\left(1+\operatorname{Diam}(T)+\log _{2}(\operatorname{Area}(D)+1)\right)$,

(iii) $\operatorname{Diam}\left(\tilde{T}^{\star}\right) \leqslant M\left(\operatorname{Diam}(T)+\operatorname{Diam}\left(T^{\star}\right)+n\right)$,

where $n:=\ell(w)$.

Proof. As $\mathcal{P}$ is fat, $\mathcal{A}$ includes some letter $z$ such that the words $z, z^{2}, z z^{-1}, z^{3}$, $z^{2} z^{-1}$ and $[a, z]$ for all $a \in \mathcal{A} \cup\{z\}$ are in $\mathcal{R}$. It will be convenient to have some further defining relators at our disposal for the construction of $\tilde{D}$. For a word $r=a_{1} a_{2} \cdots a_{p}$ define $\tilde{r}:=a_{1} z a_{2} z \cdots a_{p} z$. Then let $\tilde{\mathcal{P}}:=\langle\tilde{\mathcal{A}} \mid \tilde{\mathcal{R}}\rangle$, where $\tilde{\mathcal{A}}:=\mathcal{A}$ and $\tilde{\mathcal{R}}$ is defined to be the union of $\mathcal{R}$ with the set of words in $\left\{z, z^{-1}\right\}^{\star}$ of length four or five and with $\left\{\tilde{r} \mid r \in \mathcal{R}^{ \pm 1}\right\}$.

In what follows we show that there is a $\tilde{\mathcal{P}}$-van Kampen diagram $\tilde{D}$ for $w$ with the properties listed abovesave that the valence bound is 6 rather than 12 . Then we will show that the use of these extra nelations can $A$ circunfuente and $\bar{D}$ can be converted into a $\mathcal{P}$-van Kampen diagran for with the yalence bound increasing to no more than 12 , and without destroying the bounds (i), (i) and (i i i)

Define $C:=\max \{\ell(r) \mid r \in \tilde{\mathcal{R}}\}$, the length of the Longest of khe defining relators for $\tilde{\mathcal{P}}$.

We explain a four-step process of obtaining a $\tilde{\mathcal{P}}$-van Kampen diagram $\tilde{D}$ for $w$ from $D$. In the first three steps we invoke the methods of the electrostatic model of $\S 6$.

1. Charge the diagram $D$. We charge every 2-cell $e$ of $D$ as follows. We inscribe one 2-cell $e_{v}$ (called a charge) into $e$ at each vertex $v$ on $\partial e$. The boundary of $e_{v}$ is a single edge and its initial and terminal vertices are both identified with $v$. It is directed clockwise* and is labelled by $z$.

2. Discharge along the maximal tree $\boldsymbol{T}$. Transfer every charge to $v_{0}$ along a string of digons as described in $\S 6$.

3. Blow-up the diagram. Inflate the charges so as to produce a labelled diagram $D^{\prime}$. The inflation introduces a $z$-edge at each vertex of the boundary circuit of each of the 2-cells in $D$, changing the boundary word of each 2-cell (read anticlockwise from some vertex) from some $r \in \mathcal{R}^{ \pm 1}$ to $\tilde{r}$. Furthermore, the strings of digons inflate to give $z$-corridors along the course of geodesics in $T$ towards $v_{0}$. The effect at $v_{0}$ is to insert an extra edge path $\tau$ into the boundary circuit, so that one reads $z^{m}$ along $\tau$. Thus the boundary word is extended from $w$ to $w z^{-m}$ and $D^{\prime}$ is a $\tilde{\mathcal{P}}$-van Kampen diagram for $w z^{-m}$.

* We mean clockwise with respect to a fixed orientation of the plane and a fixed embedding of $D$ in the plane. 


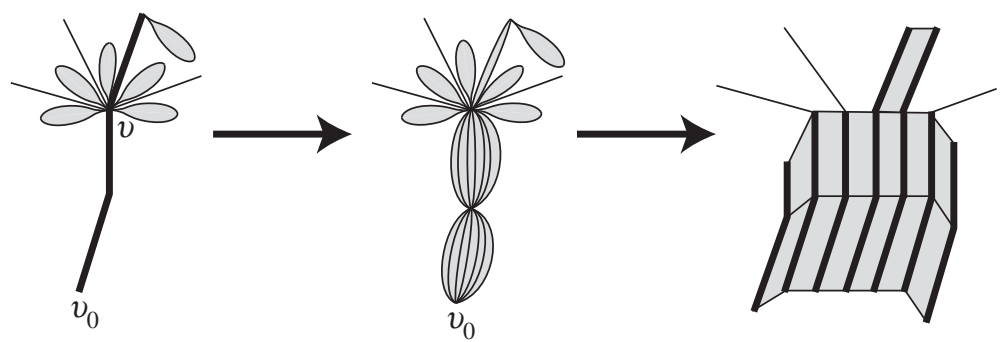

Figure 7 . The effect of charging at a vertex $v$, then discharging along a maximal tree, and then blowing up.

Notice that $m$ is the total number of charges inserted into $D$ in step 1 and

$$
m \leqslant C \text { Area }(D)
$$

because at most $C$ charges are inserted into each 2-cell of $D$.

Figure 7 illustrates the effect of charging a vertex $v$ in the interior of $D$, then discharging along a geodesic in $T$, and then blowing up. The five charges arranged around $v$ result in five $z$-edges being inserted into the boundary of the diagram at $v_{0}$. A charge at a vertex beyond $v$ in $T$ is also shown, and this contributes a sixth $z$-edge to the boundary of the diagram.

4. Attach a van Kampen diagram $B_{-r}$ reducing $z^{m}$ to the empty word. This step involves $\tilde{\mathcal{P}}$-van Kampen diagrams $B_{7 m}$ for words $z$-n (whose construction we now explain. If $m \leqslant 5$, then $B_{-m}$ is one 2-cell with $($ ( d) diredted boundary edges labelled in such a way that the boundary word is $z^{-m}$. Assume ne s bet $k$ be the least integer such that $m \leqslant 2^{k}$. The diagram $B_{-m}$ has $k-2$ concentric annular $z$-corridors, arranged around a four-sided 2-cell. All the 2-cells in the annuli are five-sided with the exception of $2^{k}-m$ in the outermost ring which are four-sided. All the edges in $B_{-m}$ are labelled by $z$. The boundary edges are directed in such a way that one reads $u$ anticlockwise around the boundary (from some starting vertex). The directions of the interior edges are chosen arbitrarily.

Figure 8 illustrates $A_{z^{-13}}$. Notice that three cells in the outermost annulus are foursided on account of $k=4$ and $2^{k}-13=3$.

We note that $B_{-m}$ is a van Kampen diagram over $\tilde{\mathcal{P}}$ and Area $\left(B_{-m}\right) \leqslant m$. Attach $B_{-m}$ to $D^{\prime}$ along $\tau$. The result is a $\tilde{\mathcal{P}}$-van Kampen diagram $\tilde{D}$ for $w$.

Now we examine the geometry of $\tilde{D}$ and prove the upper bound of 6 on the valence of its vertices. The valence of every vertex $v$ in the interior of $D^{\prime}$ after blowing up is at most 4; the effect of the blowing-up procedure on an interior vertex is illustrated in Figure 7. All vertices on $\partial D^{\prime}$ after blowing up have valence at most 3 . Vertices on $\partial B_{-m}$ also have valence at most 3 and those in the interior of $B_{-m}$ have valence at most 4 . So when $B_{-m}$ is glued onto $D^{\prime}$ along $\tau$, no vertices of valence greater than 4 are introduced, except possibly at the identified vertices $v_{1}$ and $v_{2}$ at the start and finish of the $z^{m}$ subword of $\partial D$; there is a valence 2 vertex $u$ on $\partial B_{-m}$ for all $m$, and we can assume $u$ is identified with $v_{1}$ and $v_{2}$; as $v_{1}$ and $v_{2}$ have valence at most 3 in $D^{\prime}$, the resulting vertex has valence at most 6 in $D^{\prime}$. 


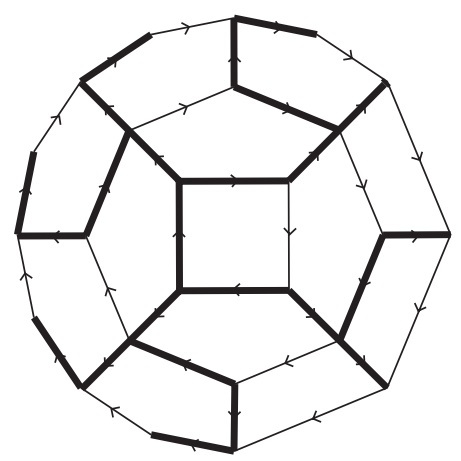

Figure 8 . The $\tilde{\mathcal{P}}$-van Kampen diagram $B_{-13}$. All edge-labels are $z$.

The bound (i) on $\operatorname{Area}(\tilde{D})$ arises from summing the following contributions.

(i) $\operatorname{Area}(D)$ 2-cells that originate in $D$.

(ii) The number of 2-cells arising from blowing up the strings of digons is at most $C$ Area $(D) \operatorname{Diam}(T)$. This is because there is one string for each charge and there are at most $C$ Area $(D)$ charges. Each string has length at most $\operatorname{Diam}(T)$.

(iii) The diagram $B$ - $n$ hes area at most $n$, which is at most GAxea $(D)$ by (7.1).

There is a natural surjection $\Phi: D^{\prime} \rightarrow D$ that collapses th $z$-corriddrs introduced in step 3. A maximal tnee $T^{\prime}$ in $\not D^{\prime}$ comprises all the odges e that have/a/single pre-image in $T$, all the sides of the $z$-corridors that were nserted in step 3. and the edge path $\tau$.

We specify a maximal tree $U$ in the interior of the diagram $B_{-m}$. Refer to the boundary components of the concentric annuli in $B_{-m}$ as rings and refer to the remaining edges not in rings as radial. We choose $U$ in such a way that it includes all the radial edges, all but one edge in the innermost ring, and alternate edges on every other ring except the outermost, in which we do not include any edges from the four-sided 2-cells. An example of $U$ is depicted with heavy lines in Figure 8.

Since $\tilde{D}$ consists of $B_{-m}$ and $D^{\prime}$ joined along $\tau$, the subgraph $\tilde{T}$ of $\tilde{D}$ that consists of all edges in $T^{\prime}$ or $U$ but not in $\tau$ is a maximal tree.

If Area $(D)=0$, then $D=\tilde{D}$ and $T=\tilde{T}$, and therefore $T^{\star}=\tilde{T}^{\star}$ consists only of the vertex at infinity. So in this case (ii) and (iii) hold with $M=1$. Assume henceforth that $\operatorname{Area}(D)>0$. It follows that $m>0$.

The maximum distance in $T^{\prime}$ of vertices from $\tau$ is at most $\operatorname{Diam}(T)$. Fix a vertex $u$ of the innermost 2-cell in $B_{-m}$. If $k$ is the least integer greater than $\log _{2} m$ then there are $(k-2) z$-annuli in $B_{-m}$ and so

$$
\operatorname{Diam}_{u}(U) \leqslant 4(k-1) \leqslant 4 \log _{2} m \leqslant 4 \log _{2}(C \text { Area }(D))
$$

the final inequality being a consequence of (7.1). So

$$
\operatorname{Diam}(\tilde{T}) \leqslant 2\left(\operatorname{Diam}(T)+4 \log _{2}(C \operatorname{Area}(D))\right) .
$$




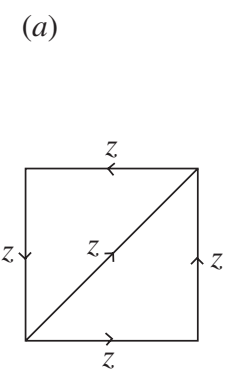

(b)

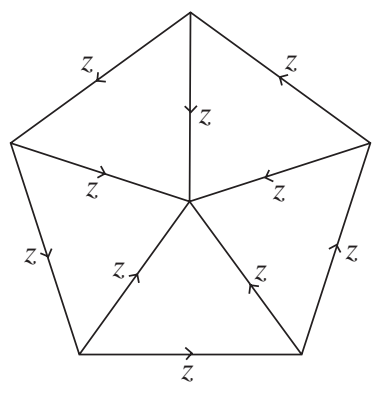

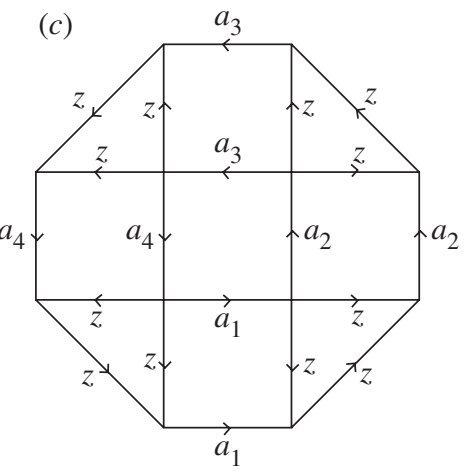

Figure 9. $\mathcal{P}$-van Kampen diagrams for $z^{4}, z^{5}$ and $a_{1} z a_{2} z a_{3} z a_{4} z$

Finally, we bound $\operatorname{Diam}\left(\tilde{T}^{\star}\right)$. From any vertex dual to a 2-cell of $B_{-m}$ in $\tilde{D}$ one can reach a vertex dual to a 2 -cell of $D^{\prime}$ within distance $k-1 \leqslant \log _{2} m \leqslant \log _{2}(C \operatorname{Area}(D))$ along a path in $\tilde{T}^{\star}$ by successively moving outwards from one annulus to the next. From any vertex dual to a 2-cell of one of the 2-cells inserted in step 3 one can reach a vertex dual one of the 2-cells originating in $D$ by following a path of length at most $\operatorname{Diam}(T)$ in $\tilde{T}^{\star}$ through a corridor. The complementary tree $T^{\star}$ of $T$ is a subtree of $\tilde{T}^{\star}$. So from any vertex dual one of the 2-cells originating in $D$ one can reach the vertex dual to the 2-cell at infinity along a path of length at most Diam $\left.T^{\star}\right)$. Therefore,

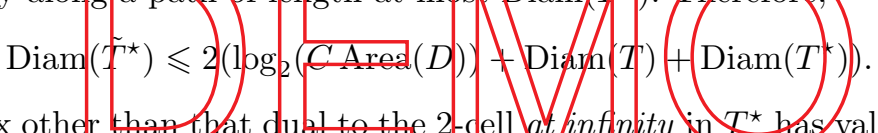

But each vertex other than that dual to the 2 -cell at inf nity in $T^{\star}$ bas valence at most $C$ and so $\operatorname{Area}(D) \leqslant n C^{\text {Dram( }}\left(T^{\star}\right)$. We deduce that $\log _{2}(C \operatorname{Area}(D)) \leqslant K \operatorname{Diam}\left(T^{\star}\right)+K n+K$ for some constant $K$, and in combination with (7.2) we have (iii).

To complete the proof we show that $\tilde{D}$ can be converted into a $\mathcal{P}$-van Kampen diagram in such a way that the bounds (i), (ii) and (iii) continue to hold (with a suitable change in the constant $M$ ) and without increasing the valence bound from 6 to more than 12 . The idea is to replace the 2 -cells with boundary words from $\tilde{\mathcal{R}} \backslash \mathcal{R}$ with $\mathcal{P}$-van Kampen diagrams as follows.

We triangulate the 2-cells with boundary words in $\left\{z, z^{-1}\right\}^{\star}$ of length four or five as illustrated in Figure $9 a, b$ in the cases $z^{4}$ and $z^{5}$. The remaining words in $\tilde{\mathcal{R}} \backslash \mathcal{R}$ are of the form $\tilde{r}=a_{1} z a_{2} z \cdots a_{p} z$ for some $r=a_{1} a_{2} \cdots a_{p}$ in $\mathcal{R}^{ \pm 1}$. We replace 2-cells with such a boundary word $\tilde{r}$ by a $\mathcal{P}$-van Kampen diagram made up of $\ell(\tilde{r})+12$-cells following the pattern of the example illustrated in Figure $9 c$. Of the 2 -cells in this diagram $\ell(\tilde{r}) / 2$ have commutators $\left[a_{i}, z\right]$ as boundary words, one has boundary word $r$, and the remaining $\ell(\tilde{r}) / 2$ have boundary words $z^{2} z^{-1}$.

In this procedure the valences of vertices are increased from at most 6 to at most 12 because in the diagrams for words in $\tilde{\mathcal{R}} \backslash \mathcal{R}$ constructed in the previous paragraph, all the boundary vertices have valence at most 3 . The area is increased by no more than a constant factor, and one can obtain a maximal tree by extending $\tilde{T}$ and in doing so one does not increase its diameter by more than an additive constant or change the diameter of the complementary tree $\tilde{T}^{\star}$ by more than a multiplicative constant. 
Lemma 7.3. Let $D$ be a diagram and $G:=D^{(1)}$. Assume that the valences of all vertices in $D$ are at most $K$. Then

$$
\operatorname{Diam}\left(G^{\star}\right) \leqslant K(\operatorname{Diam}(G)+1) .
$$

In particular, $\mathrm{GL}(D) \leqslant K(2 \operatorname{Diam}(D)+1)$.

Proof. Suppose $a^{\star}$ and $b^{\star}$ are vertices in $G^{\star}$. Let $a$ and $b$ be vertices of $G$ in the boundaries of the 2-cells dual to $a^{\star}$ and $b^{\star}$, respectively. Let $\gamma$ be a geodesic in $G$ from $a$ to $b$. The number of vertices on $\gamma$ is $(\ell(\gamma)+1) \leqslant \operatorname{Diam}(G)+1$. Let $\bar{\gamma}$ be the subdiagram of the dual diagram to $D$ consisting of the 2-cells dual to vertices on $\gamma$. There is a path in $\bar{\gamma}^{(1)}$ (and hence in $G^{\star}$ ) from $a^{\star}$ to $b^{\star}$. As the total number of edges in $\bar{\gamma}^{(1)}$ is at most $K(\operatorname{Diam}(G)+1)$, we get the required bound.

Proof of Theorem 7.1. Suppose $w$ is a length $n$ edge-circuit in $\mathcal{P}$ and that $D$ is a $\mathcal{P}$-van Kampen diagram for $w$ for which $\operatorname{Dlog} \mathrm{A}(D)=\operatorname{Dlog} \mathrm{A}(n)$. Take $T$ to be a maximal geodesic tree in $D^{(1)}$. Then by Proposition 7.2 there is a constant $M>0$, depending only on $\mathcal{P}$, such that there is a $\mathcal{P}$-van Kampen diagram $\tilde{D}$ for $w$ whose vertices have valence at most 12 and that satisfies

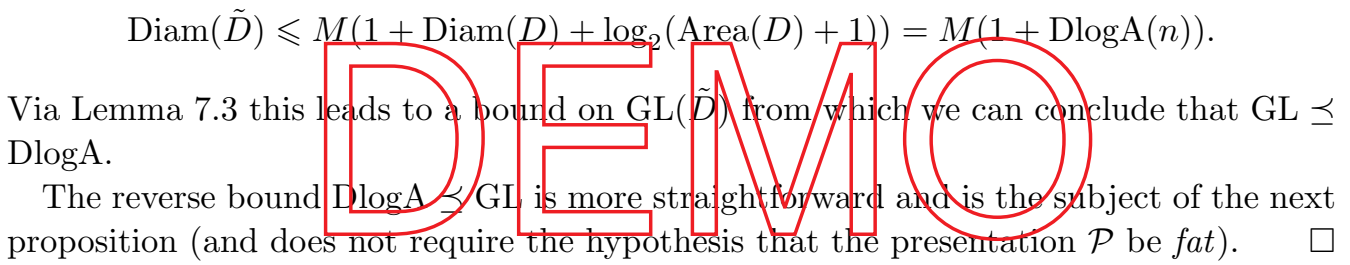

Proposition 7.4. The filling functions GL and DlogA for a finite presentation $\mathcal{P}$ satisfy $\operatorname{Dlog} \mathrm{A} \preceq$ GL.

Proof. This follows from the inequalities (1) and (5) of Proposition 2.4 in [12] since the length of the longest defining relator bounds the valence of all the vertices in the dual van Kampen diagram, with the possible exception of the vertex at infinity. Inequality (1) is used to bound the diameter term in DlogA by gallery length. Inequality (5) exploits the bounded valence of the dual graph and leads to a bound on the log-area term in DlogA by gallery length.

A further consequence of the diagram constructions of Proposition 7.2 is that in a fat finite presentation we can bound DlogA in terms of FL.

Proposition 7.5. For fat finite presentations $\mathcal{P}$ we have $\mathrm{D} \log \mathrm{A} \preceq \mathrm{FL}$.

Proof. Since FL $\simeq$ DGL for fat finite presentations by Theorem 7.1 of [12], it suffices to proves that $\operatorname{Dlog} \mathrm{A} \preceq \mathrm{DGL}$.

Let $B$ be the maximum length of the defining relators in $\mathcal{P}$. Suppose that $D$ is a van Kampen diagram for $w$ and $T$ is a maximal tree in $D^{(1)}$ for which $\operatorname{DGL}(w)=$ $\operatorname{DGL}(D)=\operatorname{Diam}(T)+\operatorname{Diam}\left(T^{\star}\right)$. Then for the diagram $\tilde{D}$ of Proposition 7.2 we 
can use (iii) to give $\operatorname{Diam}\left(\tilde{T}^{\star}\right) \leqslant M(\operatorname{DGL}(D)+n)$, where $n:=\ell(w)$. It follows that $\operatorname{Diam}(\tilde{D}) \leqslant B M(\operatorname{DGL}(D)+n)+n / 2$.

Now the area of $\tilde{D}$ is at most $M \operatorname{Area}(D)(1+\operatorname{Diam}(T))$ by (i). But

$$
\operatorname{Area}(D) \leqslant n(B-1)^{\operatorname{Diam}\left(T^{\star}\right)}
$$

by (5) of Proposition 2.4 of $[\mathbf{1 2}]$. So $\log _{2}(1+\operatorname{Area}(\tilde{D})) \leqslant K \operatorname{DGL}(D)+K n+K$ for some constant $K>0$. Deduce that for some constant $K^{\prime}>0$,

$$
\operatorname{Dlog} \mathrm{A}(D)=\operatorname{Diam}(\tilde{D})+\log _{2}(1+\operatorname{Area}(\tilde{D})) \leqslant K^{\prime} \operatorname{DGL}(D)+K^{\prime} n+K^{\prime} .
$$

So $\operatorname{Dlog} \mathrm{A} \preceq \mathrm{DGL}$.

\section{Estimates on gallery length and filling length}

A first application of Theorem 7.1 is a concise proof of the second half of the following result.

Theorem 8.1. Suppose $\mathcal{P}$ and $\mathcal{Q}$ are finite presentations for quasi-isometric groups. Then $\mathrm{FL}_{\mathcal{P}} \simeq \mathrm{FL}_{\mathcal{Q}}$. If, in addition, $\mathcal{P}$ and $\mathcal{Q}$ are both fat, then $\mathrm{GL}_{\mathcal{P}} \simeq \mathrm{GL}_{\mathcal{Q}}$.

Proof. We will biefty recall the well-knomp proof 1 that Area is a quasi-isometry invariant of finitely presented proups up to f-dquivaldnce, and we will then adapt it to prove our theorem.

Define $F: C(\mathcal{P})^{(0)} \rightarrow C(2)(0)$ td be a quasi-isondtry rom the o sleleton of $C(\mathcal{P})$ to that of $C(\mathcal{Q})$ and let $G: C(\mathcal{Q})^{(0)} \rightarrow C(\mathcal{P})^{(0)}$ be a quasi-inverse for $F$. Suppose $w$ is an edge-circuit in $C(\mathcal{P})$. Join the images under $F$ of adjacent vertices of $w$ by geodesics to form an edge-circuit $\hat{w}$ in $C(\mathcal{Q})$. Given a van Kampen diagram $\Phi: \hat{D} \rightarrow C(\mathcal{Q})$ for $\hat{w}$, use $\left.G \circ \Phi\right|_{\hat{D}^{(0)}}$ to map the vertices of $\hat{D}$ to $C(\mathcal{P})^{(0)}$. Joining the images of end points of edges in $\hat{D}$ by geodesics, and then filling the faces with minimal area diagrams makes a van Kampen diagram $\bar{D} \rightarrow C(\mathcal{P})$ for an edge-circuit $\bar{w}$ in $C(\mathcal{P})$. An annular diagram $A$ of bounded width is then attached to the boundary of $\bar{D}$ to make a van Kampen diagram $D$ for $w$. Comparing the area of $\hat{D}$ with that of $D$ leads to the proof that Area is a quasi-isometry invariant.

To show that FL is a quasi-isometry invariant, one induces a shelling of $D$ from a shelling of $\hat{D}$ in the way we now sketch. Shell $D$ by first radially shelling the annulus $A$, leaving only a path $p$ (of length depending only on $\mathcal{P}$ and $\mathcal{Q}$ ) from the base point of $D$ to $\bar{D}$, then shell $\bar{D}$ mimicking a shelling of $\hat{D}$, and finally shell $p$.

Similarly, the methods of [1] can be extended to (Area,Diam)-pairs. Thus one sees DlogA to be a quasi-isometry invariant. We then use Theorem 7.1 to show that GL is a quasi-isometry invariant of finite fat presentations up to equivalence, which completes the argument.

Theorem 7.1 is used to calculate upper bounds on gallery length in the following theorem. 


\section{Theorem 8.2.}

(i) The gallery length function of any finite presentation of a group admitting a polynomial isoperimetric inequality of degree $d \geqslant 2$ admits a polynomial upper bound of degree $d-1$.

(ii) The gallery length function of the presentation $\langle x, y, s, t|[x, y]=1, t x t^{-1}=$ $x^{2}$, sys $\left.^{-1}=y^{2}\right\rangle$, due to Bridson, admits a linear upper bound.

If Conjecture 5.4 holds, then the filling length functions admit the same upper bounds.

Proof. For (i) and (ii) we apply Theorem 7.1 to the (Area, Diam)-pairs $\left(n^{d}, n^{d-1}\right)$ and $\left(E^{n}, n\right)$ for $\mathcal{P}$ (up to common multiplicative constants), respectively. This (Area, Diam)pair for (i) is [10, Theorem 2] and that for (ii) is a consequence of the computations in $\S 5$ of $[\mathbf{1 1}]$.

The coda follows from Theorem 1.3.

In the context of Theorem 8.2(i) we mention that it is proved in [20, Corollary 5.5] that finitely presented groups that admit quadratic isoperimetric functions also admit linear upper bounds on their filling length functions. This adds credence to Conjecture 5.4. We also note that in the case when $d=2$, the linear bound of Theorem 8.2(i) applies in particular to Thompson's greup $F$ on account of the recent result of Guba [16].

Theorem 8.3. Asynchronptusly combable go beps hade filling length functions admitting linear upper bounds. Thys inclides

(i) fundamental groups of hnite graphs ot gioups whth Gihitely generated free vertex and edge groups (for example, the Baumslag-Solitar groups $\mathrm{BS}(p, q)=\langle x, y| y^{-1} x^{p} y=$ $\left.\left.x^{q}\right\rangle\right)$;

(ii) fundamental groups of compact, geometrizable 3-manifolds;

(iii) split extensions of hyperbolic or abelian groups by asynchronously combable groups.

Proof. It is a result of the first author, expressed with the notation $L N C H_{1}$ in $[\mathbf{9}$, Theorem 3.1], that the filling length functions of asynchronously combable groups admit linear upper bounds.

That the groups listed are asynchronously combable follows from Theorem $\mathrm{F}$ and Corollary E1 of [2] for (i) and from Bridson [3] for (ii) and (iii).

Finitely generated nilpotent groups are also known to have filling length functions that admit linear upper bounds [13-15]. It is an open question whether or not such groups are asynchronously combable.

The filling length functions of groups with presentations that are almost convex in the sense of Cannon also admit linear upper bounds as we will now explain. Recall that a finite presentation $\mathcal{P}$ satisfies Cannon's almost convexity condition $\mathrm{AC}(2)$ when there exist integers $K$ and $n_{0}$ with the following properties. For all vertices $a, b$ in the Cayley graph $C(\mathcal{P})^{(1)}$ of $\mathcal{P}$, at an equal distance $n:=d(1, a)=d(1, b)$ from the identity, if 
$d(a, b) \leqslant 2$ and $n \geqslant n_{0}$, then there is an edge-path from $a$ to $b$ of length at most $K$ that is contained in the closed ball $B_{n}(1)$ of radius $n$ about the identity. (There are also conditions $\mathrm{AC}(k)$ for $k \geqslant 2$, in which $d(a, b)$ is allowed to be at most $k$ rather than at most 2. The proof of the theorem can easily be generalized to apply to $\mathrm{AC}(k)$ presentations.)

Theorem 8.4. The filling length function of any group $\Gamma$ that has a finite presentation $\mathcal{P}=\langle\mathcal{A} \mid \mathcal{R}\rangle$ satisfying Cannon's almost convexity condition $\mathrm{AC}(2)$ admits a linear upper bound.

Before proving this theorem we adapt a definition of Gromov [14, p. 101] to the combinatorial setting of a Cayley 2-complex.

Definition 8.5. Let $\mathcal{P}$ be a finite presentation of a group $\Gamma$. Suppose $w_{1}, w_{2} \in\left(\mathcal{A}^{ \pm 1}\right)^{\star}$ are words with $w_{1}=w_{2}$ in $\Gamma$ and that $D$ is a van Kampen diagram with boundary word $w_{2} w_{1}^{-1}$, read anticlockwise from a base vertex $v_{1}$. Let $v_{2}$ be the vertex of the boundary circuit at the end of the word $w_{2}$, as one reads from $v_{1}$. (So $v_{1}=v_{2}$ precisely when either $w_{1}$ or $w_{2}$ is the empty word.)

Roughly speaking, we define $\mathrm{F}_{+} \mathrm{L}\left(w_{1}, w_{2}, D\right)$ to be the minimal length $L$ such that there is a combinatorial homotopy of $w_{1}$ to $w_{2}$ across $D$ through paths of length at most $L$ that have fixed end points $v_{1}$ and $v_{2}$. Formally, a combinatorial homotopy of $w_{1}$ to $w_{2}$ across $D$ is a sequence of van Kampen diagrams $\mathcal{H}=\left(D_{1}, D_{2}, \ldots, D_{m}\right)$ such that $D_{1}=D$ and $D_{m}$ is a simple edge path aleng which one reads yz; agch $D_{i+1}$ is obtained from $D_{i}$ by either a 1 cell colapse or a 1-cell dxpansidn. of 2-cely dollapse move (see Definition 2.3 in [12) in such a way that the $u_{2}$ porthon of the boundary words $\partial D_{i}$ is never broken.* Define

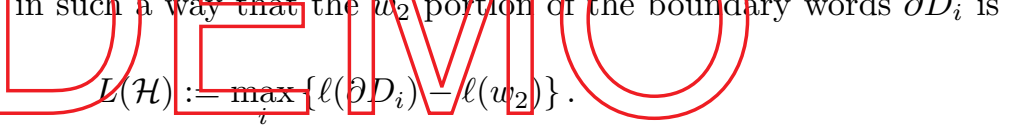

Then $\mathrm{F}_{+} \mathrm{L}\left(w_{1}, w_{2}, D\right)$ is the minimum of $L(\mathcal{H})$ among all combinatorial homotopies $\mathcal{H}$ of $w_{1}$ to $w_{2}$ across $D$. Define

$$
\mathrm{F}_{+} \mathrm{L}\left(w_{1}, w_{2}\right):=\min \left\{\mathrm{F}_{+} \mathrm{L}\left(w_{1}, w_{2}, D\right) \mid \operatorname{van} \text { Kampen diagrams } D \text { for } w_{2} w_{1}{ }^{-1}\right\} .
$$

Finally, we define a function $\mathrm{F}_{+} \mathrm{L}: \mathbb{N} \rightarrow \mathbb{N}$ by

$$
\mathrm{F}_{+} \mathrm{L}(n):=\max \left\{\mathrm{F}_{+} \mathrm{L}\left(w_{1}, w_{2}\right) \mid \text { words } w_{1}, w_{2} \text { with } \ell\left(w_{1}\right), \ell\left(w_{2}\right) \leqslant n \text { and } w_{1}={ }_{\Gamma} w_{2}\right\} .
$$

The observation that a combinatorial homotopy $\mathcal{H}$ of $w_{1}$ to $w_{2}$ across a van Kampen diagram $D$ can be extended to a shelling of $D$ down to the base vertex $v_{1}$ leads to the following proposition.

Proposition 8.6. The functions $\mathrm{FL}$ and $\mathrm{F}_{+} \mathrm{L}$ for an arbitrary finite presentation are related as follows. For all $n$,

$$
\begin{aligned}
\mathrm{FL}(2 n) & \leqslant \mathrm{F}_{+} \mathrm{L}(n), \\
\mathrm{FL}(2 n+1) & \leqslant \mathrm{F}_{+} \mathrm{L}(n+1) .
\end{aligned}
$$

In particular, if $\mathrm{F}_{+} \mathrm{L}$ admits a linear upper bound, then so does FL.

* That is, the word one reads anticlockwise around the boundary of $D_{i}$ starting from the base vertex $v_{1}$ begins with an edge-path $q$ along which one reads $w_{2}$, and each 1-cell or 2-cell collapse move $D_{i} \rightarrow D_{i+1}$ does not collapse an edge of $q$ and each 1-cell collapse move inserts two edges in such a way that an inverse pair is inserted into the boundary word somewhere after the prefix word $w_{2}$. 

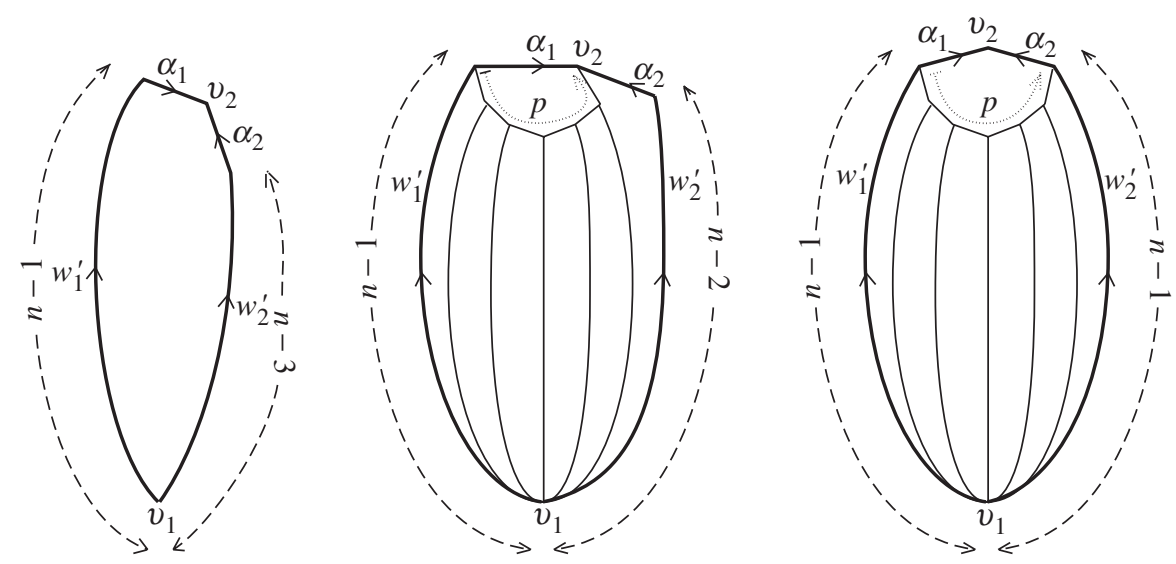

Figure 10. The three cases $\ell\left(w_{2}^{\prime}\right)=n-3, n-2, n-1$.

Proof of Theorem 8.4. It will suffice to show that for $n \geqslant n_{0}+1$,

$$
\mathrm{F}_{+} \mathrm{L}(n) \leqslant \mathrm{F}_{+} \mathrm{L}(n-1)+1+C,
$$

where $C$ is a constant depending only on $\mathcal{P}$, for then it will follow that $\mathrm{F}_{+} \mathrm{L}(n)$ admits a linear bound, and thereforese does FL $(n)$ by Proposition 8.6

Fix $n \geqslant n_{0}+1$ Suppose thet $w_{1}, w_{2} \&\left(\mathcal{A}^{ \pm}\right)^{*}$ are two wprds with lengths $\ell\left(w_{1}\right), \ell\left(w_{2}\right) \leqslant n \operatorname{such}$ that $\left.w_{1}\right)={ }_{\Gamma} w_{2}$. We will show that

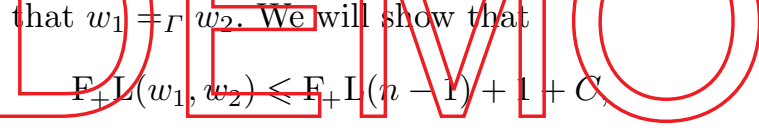

where $C:=\max \left\{\mathrm{F}_{+} \mathrm{L}(\max \{1, K\}), \mathrm{F}_{+} \mathrm{L}(1+K)\right\}$.

We assume that either $\ell\left(w_{1}\right)=n$ or $\ell\left(w_{2}\right)=n$, for otherwise $\mathrm{F}_{+} \mathrm{L}\left(w_{1}, w_{2}\right) \leqslant \mathrm{F}_{+} \mathrm{L}(n-1)$ and (8.1) is immediately satisfied.

Case $\ell\left(\boldsymbol{w}_{\mathbf{1}}\right)=\boldsymbol{n}$. For $i=1,2$ write $w_{i}=w_{i}^{\prime} \alpha_{i}$, where $\alpha_{i} \in \mathcal{A}^{ \pm 1}$, and let $w_{i}^{\prime \prime}$ be a geodesic word with $w_{i}^{\prime}={ }_{\Gamma} w_{i}^{\prime \prime}$. Then

$$
\begin{aligned}
\mathrm{F}_{+} \mathrm{L}\left(w_{1}, w_{2}\right) & =\mathrm{F}_{+} \mathrm{L}\left(w_{1}^{\prime} \alpha_{1}, w_{2}^{\prime} \alpha_{2}\right) \\
& \leqslant \max \left\{\mathrm{F}_{+} \mathrm{L}\left(w_{1}^{\prime} \alpha_{1}, w_{1}^{\prime \prime} \alpha_{1}\right), \mathrm{F}_{+} \mathrm{L}\left(w_{1}^{\prime \prime} \alpha_{1}, w_{2}^{\prime \prime} \alpha_{2}\right), \mathrm{F}_{+} \mathrm{L}\left(w_{2}^{\prime \prime} \alpha_{2}, w_{2}^{\prime} \alpha_{2}\right)\right\} \\
& \leqslant \max \left\{1+\mathrm{F}_{+} \mathrm{L}\left(w_{1}^{\prime}, w_{1}^{\prime \prime}\right), \mathrm{F}_{+} \mathrm{L}\left(w_{1}^{\prime \prime} \alpha_{1}, w_{2}^{\prime \prime} \alpha_{2}\right), 1+\mathrm{F}_{+} \mathrm{L}\left(w_{2}^{\prime \prime}, w_{2}^{\prime}\right)\right\} \\
& \leqslant \max \left\{1+\mathrm{F}_{+} \mathrm{L}(n-1), \mathrm{F}_{+} \mathrm{L}\left(w_{1}^{\prime \prime} \alpha_{1}, w_{2}^{\prime \prime} \alpha_{2}\right)\right\} .
\end{aligned}
$$

So we may, in fact, assume that $w_{1}^{\prime}$ and $w_{2}^{\prime}$ are geodesic words, and then, as $\ell\left(w_{1}^{\prime}\right)=n-1$, we find $\ell\left(w_{2}^{\prime}\right) \in\{n-3, n-2, n-1\}$. These three eventualities are illustrated in Figure 10 and we will address each one in turn.

Subcase $\ell\left(w_{2}^{\prime}\right)=n-3$. We have

$$
\mathrm{F}_{+} \mathrm{L}\left(w_{1}, w_{2}\right) \leqslant \mathrm{F}_{+} \mathrm{L}\left(w_{1}^{\prime}, w_{2}^{\prime} \alpha_{2} \alpha_{1}^{-1}\right) \leqslant \mathrm{F}_{+} \mathrm{L}(n-1),
$$

and (8.1) is satisfied. 
Subcase $\ell\left(w_{2}^{\prime}\right)=n-2$. The length of $w_{2}$ is $n-1$ and we may assume it to be a geodesic word (for it is possible to combinatorially homotop $w_{2}$ to any word $\hat{w}_{2}$ with length at most $n-1$ and with $w_{2}={ }_{\Gamma} \hat{w}_{2}$ through paths of length at most $\left.\mathrm{F}_{+} \mathrm{L}(n-1)\right)$.

Let $D$ be a diagram consisting of one 2-cell with boundary circuit of $\ell\left(w_{1}\right)+\ell\left(w_{2}\right)$ edges, and let $\Phi: D^{(1)} \rightarrow C(\mathcal{P})$ be a combinatorial map with image the edge-circuit $w_{2} w_{1}{ }^{-1}$ based at the identity $\Phi\left(v_{1}\right)=1$. As $n-1 \geqslant n_{0}$, the $\mathrm{AC}(2)$ condition allows us to extend $\Phi$ to a map $\hat{\Phi}: \hat{D}^{(1)} \rightarrow C(\mathcal{P})$, where $\hat{D}$ is Figure $10 b$; the image $\hat{\Phi}(p)$ of the path $p$ shown is an edge path in $B_{n-1}(1)$ of length at most $K$, and $\hat{\Phi}$ maps each of the edge paths that run from $v_{1}$ to vertices on $p$ (depicted almost vertically) to geodesic in $C(\mathcal{P})$.

We combinatorially homotop $w_{1}$ to $w_{2}$ through paths of lengths within the bound (8.1) as follows. We first homotop $\alpha_{1}$ to $p$ across some van Kampen diagram filling the uppermost 2-cell of the diagram. As $\ell(p) \leqslant K$ this can be done through paths of length at most $\mathrm{F}_{+} \mathrm{L}(\max \{1, K\}) \leqslant C$. Next we homotop across van Kampen diagrams filling each of the vertical 2-cells in turn working from left to right (in the sense of the depiction of the diagram in Figure 10) as follows.

If $u_{1}$ and $u_{2}$ are the initial and terminal vertices of an edge $e$ of $p$, then either $d\left(\hat{\Phi}\left(u_{1}\right), \hat{\Phi}\left(v_{1}\right)\right) \leqslant n-2$ or $d\left(\hat{\Phi}\left(u_{2}\right), \hat{\Phi}\left(v_{1}\right)\right) \leqslant n-2$, for otherwise the image of the midpoint of $e$ would be outside $B_{n-1}(1)$. Homotop through paths of length at most $\mathrm{F}_{+} \mathrm{L}(n-1)$ with fixed end points $u_{2}$ and $v_{1}$ if $d\left(\hat{\Phi}\left(u_{1}\right), \hat{\Phi}\left(v_{1}\right)\right) \leqslant n-2$ and fixed end points $u_{1}$ and $v_{1}$ otherwise. In the pecond gase we next dollappe $e$.

Subcase $\ell\left(w_{2}^{\prime}\right)=n-1$. Th s is imilar to the previdus pupqase. The images of the end vertices of $w_{1}^{\prime}$ and $u_{2}^{\prime}$ are manped by $\hat{\Phi}$ to points al distande $n-1$ from 1 in $C(\mathcal{P})^{(1)}$,

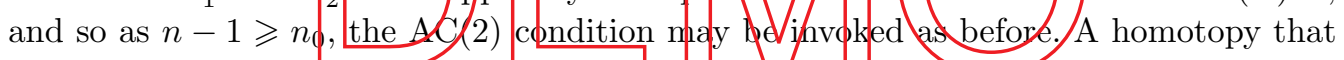
satisfies (8.1) begins with a homotopy of $\alpha_{1}$ to $p \alpha_{2}$, and continues with homotopies across the vertical 2-cells proceeding from left to right.

Case $\boldsymbol{\ell}\left(\boldsymbol{w}_{\mathbf{2}}\right)=\boldsymbol{n}$. The proof is similar to the case $\ell\left(w_{1}\right)=n$. The problem is reduced to considering the three subcases $\ell\left(w_{1}^{\prime}\right)=n-3, n-2, n-1$ and diagrams that are reflections of those in Figure 10 are examined. So it suffices to note that the minimal length $L$ of paths in a right-to-left combinatorial homotopy across the diagrams depicted is within the bound (8.1).

Groups with presentations satisfying various weaker forms of the almost convexity condition are also studied in the literature and their filling length functions are known to admit quadratic upper bounds [19, Theorem 1].

The results listed above suggest a prevalence of finitely presented groups whose gallery length functions admit linear upper bounds and begs the question of whether there exist finite presentations whose gallery length functions or filling length functions grow faster. The answer is that there are many such groups. As we mentioned in $\S 5$, the word problem for a finite presentation $\mathcal{P}$ is solvable if and only if one of $\mathrm{GL}_{\mathcal{P}}$ or $\mathrm{FL}_{\mathcal{P}}$ (and hence both) is bounded above by a recursive function. It follows that finite presentations $\mathcal{P}$ for groups with unsolvable word problem have $\mathrm{GL}_{\mathcal{P}}$ and $\mathrm{FL}_{\mathcal{P}}$ both growing faster than any recursive function. Also the family

$$
\Gamma_{n}=\left\langle x_{0}, \ldots x_{n} \mid x_{0}{ }^{-1} x_{1} x_{0}=x_{1}{ }^{2}, \ldots, x_{n-1}{ }^{-1} x_{n} x_{n-1}=x_{n}{ }^{2}\right\rangle
$$


$\left[14, \S 4 . \mathrm{C}_{3}\right]$ of groups that have Dehn functions $\simeq$-equivalent to an $n$-times iterated exponential function have filling length and gallery length functions growing at least as fast as an $(n-1)$-times iterated exponential function on account of the inequalities relating $\operatorname{Area}(n)$ to $\mathrm{GL}(n)$ and $\operatorname{Area}(n)$ to $\mathrm{FL}(n)$ discussed in $\S 5$ of [12]. Bridson's presentations [4] for groups $\Phi_{m}$ defined for $m \geqslant 2$ by

$$
\begin{aligned}
\left\langle a_{1}, \ldots, a_{m}, s, t, \tau\right| \text { for } i<m, s^{-1} a_{i} s & =a_{i} a_{i+1}, \\
{\left[t, a_{i}\right]=\left[\tau, a_{i}\right] } & \left.=\left[s, a_{m}\right]=\left[t, a_{m}\right]=\left[\tau, a_{m} t\right]=1\right\rangle
\end{aligned}
$$

are contrasting examples. Bridson proves that these have minimal isodiametric functions $\operatorname{Diam}_{\Phi_{m}}(n) \simeq n^{m}$ and have Dehn functions $\operatorname{Area}_{\Phi_{m}}(n) \simeq n^{2 m+1}$. Their filling length and gallery length functions lie between $\operatorname{Diam}_{\Phi_{m}}(n)$ and $\operatorname{Area}_{\Phi_{m}}(n)$. (This follows from $[\mathbf{1 2}$, Proposition 2.4].)

However, one can speculate about uniform upper bounds on Diam, FL, GL and Area for particular classes of groups. In this context we mention a conjecture and a question of the first author. The conjecture has been in the public domain for a while but, to our knowledge, is set down in print for the first time here.

Conjecture 8.7. There is a common recursive upper bound for the Dehn functions of all finite presentations of linear groups.

Here, by a common upper Dound, we mean functidn $f: \mathbb{N} \rightarrow$ such that all the Dehn functions are $\preceq f(n)$ in the sense of $\mathbb{D}$ ef nition 5.3 . The authors are unaware of any finitely presented lineas group with Dehn function or ow ng faster than exponential. One might begin by trying to answer the following.

Question 8.8. Do the filling length functions of all finite presentations of polycyclic groups admit linear upper bounds?

A positive answer is already known in the special case of nilpotent groups (see $[\mathbf{1 3}$, Corollary B.1], [20]) and would imply exponential isoperimetric functions for the presentations in question (by $[\mathbf{1 0}$, Corollary 2] or $[\mathbf{1 4}, 5 . \mathrm{C}]$ ).

\section{Relationships between filling functions}

We summarize known relationships between filling functions in the following theorem.

Theorem 9.1. For a finite presentation $\mathcal{P}$,

(a) Diam $\preceq$ DlogA, GL, FL, DGL $\preceq$ Area,

(b) $\mathrm{D} \log \mathrm{A} \preceq \mathrm{GL}$,

(c) $\log (1+$ Area $) \preceq$ FL, GL,

(d) $\mathrm{FL} \preceq \mathrm{D} \log \mathrm{A}^{2}$,

(e) $\log (1+\mathrm{FL}), \log (1+\mathrm{GL}) \preceq$ Diam, 
(f) $\log \log (1+$ Area $) \preceq$ Diam.

Moreover, if $\mathcal{P}$ is fat, then

(g) $\mathrm{GL} \simeq \mathrm{D} \log \mathrm{A} \preceq \mathrm{FL} \simeq \mathrm{DGL}$.

If, in addition, Conjecture 4.3 holds, then

(h) $\mathrm{GL} \simeq \mathrm{D} \log \mathrm{A} \simeq \mathrm{FL} \simeq \mathrm{DGL}$.

Proof. The bounds on Diam by DlogA and DGL in (a) are immediate from the definitions, and the bounds by GL and FL follow from (1), (2) of Proposition 2.4 in [12]. That GL, DlogA, FL, DGL $\preceq$ Area follows from (3), (4) of Proposition 2.4 in [12] and the easy result that $\operatorname{Diam}(D) \preceq B \operatorname{Area}(D)+\operatorname{Perimeter}(D)$ for a diagram $D$ whose 2-cells all have at most $B$ boundary edges.

We proved (b) in Proposition 7.4. The bound $\log (1+$ Area $) \preceq \mathrm{FL}$ of $(c)$ is the spacetime bound of $[\mathbf{1 0}$, Corollary 2] or $[\mathbf{1 4}, 5 . \mathrm{C}]$. The corresponding bound for GL follows from [12, Proposition 2.4 (5)]. Theorem 1 of [10] implies (d).

The bound $\log (1+\mathrm{GL}) \preceq$ Diam of (e) follows from [12, Theorem 5.1]. The double exponential theorem, a recent new proof of which is in $\S 5$ of $[\mathbf{1 2}]$ (cf. references therein), gives (f). The other bound $\log (1+\mathrm{FL}) \preceq \operatorname{Diam}$ of $(\mathrm{e})$ is pbtained combining the double exponential theorem with Theorem 1 of $[\mathbf{1 0}]$

It remains to prove $(\mathrm{g})$ and $(\mathrm{h})$. When $\mathcal{P}$ is a fat presentation we get $\mathrm{dL} \simeq \operatorname{Dlog} \mathrm{A}$ from Theorem 7.1. The equivalence EL $\simeq$ DGL is theoren 7 .1 of [12]. The result DlogA $\preceq$ FL is Proposition 7.5 above. Il Conjecture $5.4 \mathrm{~h}$. ds, then by Theorem 1.3 these four filling functions are all $\simeq$-equivalent.

\section{Restricted filling functions}

One of the features of the van Kampen diagrams $\tilde{D}$ for null-homotopic words $w$ in a fat presentation $\mathcal{P}$ constructed in Proposition 7.2 is that they have uniformly bounded valence. Remark 7.3 in [12] explains that the same is true of the diagrams $\bar{D}$ constructed in the proof of Theorem 7.1 in [12]. Thus by adding a, in one sense, redundant extra generator $z$ and some apparently innocuous extra defining relators to a finite presentation, we have ensured that every edge-circuit admits a van Kampen diagram in which every vertex valence is at most 12. Moreover, both in Proposition 7.2 and in Theorem 7.1 in [12] we have substantial control on the geometry of that diagram. (Several consequences will be included in Theorem 10.3.) This motivates us to define what we call restricted filling functions, where we quantify over all diagrams whose vertices have valence within some specified bounded.

Definition 10.1. Suppose that $\mathcal{P}$ is a finite presentation and that $\mathcal{F}$ is a diagram measurement (see Definition 4.2). Fix $k>0$. Suppose $w$ is an edge-circuit in $\mathcal{P}$. If $w$ fails to admit a van Kampen diagram in which the valence of every vertex is at most $k$, then $R_{k} \mathcal{F}(w):=\infty$. Otherwise we define $R_{k} \mathcal{F}(w)$ to be the minimum of $\mathcal{F}(D)$ over all 
van Kampen diagrams $D$ for $w$ in which every vertex has valence at most $k$. Then we define the restricted filling function $R_{k} \mathcal{F}: \mathbb{N} \rightarrow \mathbb{N} \cup\{\infty\}$ by

$$
R_{k} \mathcal{F}(n):=\sup \left\{R_{k} \mathcal{F}(w) \mid \text { edge-circuits } w \text { with } \ell(w) \leqslant n\right\} .
$$

The bounded valence of the diagrams in Proposition 7.2 implies the following. (It is likely that the bound of 12 can be improved.)

Proposition 10.2. If $\mathcal{P}$ is a finite fat presentation and $\mathcal{F}$ is any diagram measurement, then $R_{12} \mathcal{F}(n)<\infty$ for all $n$.

Restricted filling functions have geometric significance in the realm of combinatorial notions of curvature. Suppose we give the corners* of the 2-cells in the presentation 2 -complex $\mathcal{K}^{2}$ (see $\S 5$ ) strictly positive weights (i.e. angles), then the corners of 2-cells in van Kampen diagrams inherit weights (via the map $\Phi$ (see Definition 5.1)). Then restricted filling functions concern the geometry of diagrams with curvature uniformly bounded away from $-\infty$; that is, diagrams for which there is a uniform bound on the sum of the weights at each corner.

In the following theorem we set out some of the properties of the restricted forms of the filling functions we have been discussing.

Theorem 10.3. Let $\mathcal{P}$ be a fat finite presenfation. Fix $k \geqslant 12$ and define $R:=R_{k}$.

(a) If $\mathcal{F}$ is a filling function tor $P$, then $\mathcal{F} \npreceq R \mathcal{F}$.

(b) If $(f, g)$ is an (Area,Dane)-pair, then $f f g .1\left(f g+\log _{2}(1+f)\right)$ is an ( $R$ Area, $R$ Dian)-pair for $\mathcal{P}$, up to a commdn mult plicative constant.

(c) $\mathrm{FL} \simeq R \mathrm{FL} \simeq \mathrm{DGL} \simeq R \mathrm{DGL}$ and $R \mathrm{D} \log \mathrm{A} \simeq \mathrm{D} \log \mathrm{A}$

(d) $R$ Diam $\simeq R \mathrm{GL} \simeq \operatorname{DlogA}$.

Moreover, if Conjecture 4.3 is true, then $R \mathrm{GL} \simeq R \mathrm{DGL}$ and so all the functions from (c) and (d) are $\simeq$-equivalent.

Proof. The explanation for (a) is that for an edge-circuit $w$, in the definition of $R \mathcal{F}(w)$ one quantifies over a (non-empty) subset of the van Kampen diagrams that one quantifies over in the definition of $\mathcal{F}(w)$.

To prove (b) use Proposition 7.2 with $T$ a geodesic maximal tree in $D^{(1)}$. The diagram $\tilde{D}$ has area at most $M(f(n)+f(n) g(n))$ by (i) and diameter at most $M(1+g(n)+$ $\left.\log _{2}(1+f(n))\right)$ by (ii).

For $R \mathrm{DGL} \simeq R \mathrm{FL}$ we note that $R \mathrm{FL} \preceq R \mathrm{DGL}$ is immediate from the inequality relating the diagram measurements FL and DGL in Theorem 3.5 of [12]. The reverse inequality $R \mathrm{DGL} \preceq R \mathrm{FL}$ is a consequence of the constructions used to show DGL $\preceq \mathrm{FL}$ in the proof of Theorem 7.1 in $[\mathbf{1 2}]$.

We learn from (a) that it suffices to show that $R \mathrm{FL} \preceq \mathrm{FL}$ and $R \mathrm{D} \log \mathrm{A} \preceq \mathrm{D} \log \mathrm{A}$ in order to establish the remaining equivalences in (c).

* A corner of a 2-cell is one of the subdivision points of its attaching map. 
We know from (a) that FL $\preceq R$ FL and DGL $\preceq R$ DGL. In the following paragraph we prove that $R \mathrm{FL} \preceq \mathrm{DGL}$ and then in the next paragraph we explain $R \mathrm{DGL} \preceq$ FL. We can then deduce that FL, $R \mathrm{FL}, \mathrm{DGL}$ and $R \mathrm{DGL}$ are all $\simeq$-equivalent.

Suppose $w$ is an edge-circuit in $\mathcal{P}$. Suppose that $D$ is a van Kampen diagram for $w$ and $T$ is a maximal tree in $D^{(1)}$ for which $\operatorname{DGL}(w)=\operatorname{DGL}(D)=\operatorname{Diam}(T)+\operatorname{Diam}\left(T^{\star}\right)$. Then for the diagram $\tilde{D}$ of Proposition 7.2 we can use (iii) to bound $\operatorname{Diam}\left(\tilde{T}^{\star}\right)$ in terms of $\operatorname{DGL}(D)$. We bound the $\log _{2}\left(\operatorname{Area}(D)+1\right.$ ) term in (ii) using $\operatorname{Diam}\left(T^{\star}\right)$ (see $[\mathbf{1 2}$, Proposition $2.4(5)]$ ), and the result is $\operatorname{Diam}(\tilde{T})$ majorized in terms of $\operatorname{DGL}(D)$. As vertex valences in $\tilde{D}$ are at most 12 we deduce that $R \mathrm{FL} \preceq$ DGL.

In $\S 7$ of $[\mathbf{1 2}]$ we proved that for a fat presentation $\mathcal{P}$ one has DGL $\preceq$ FL. Given a null-homotopic word $w$ we took a minimal filling length van Kampen diagram $D$ for $w$ and we constructed a new van Kampen diagram $\bar{D}$ for $w$ for which $\operatorname{DGL}(\bar{D})$ could be bound in terms of $\mathrm{FL}(D)$. As noted in Remark 7.3 of that article, all vertices in the diagram $\bar{D}$ had valences at most 11 . So, in fact, our proof amounted to showing that $R \mathrm{DGL} \preceq \mathrm{FL}$.

To show $R \mathrm{D} \log \mathrm{A} \preceq \mathrm{D} \log \mathrm{A}$ we suppose that $D$ is a van Kampen diagram for an edgecircuit $w$ for which $\operatorname{Dlog} \mathrm{A}(D)=\operatorname{Dlog} \mathrm{A}(w)$. Then the diagram $\tilde{D}$ of Proposition 7.2, with $T$ a geodesic maximal tree in $D^{(1)}$, has all vertex valences at most 12 and by (i), (ii), respectively,

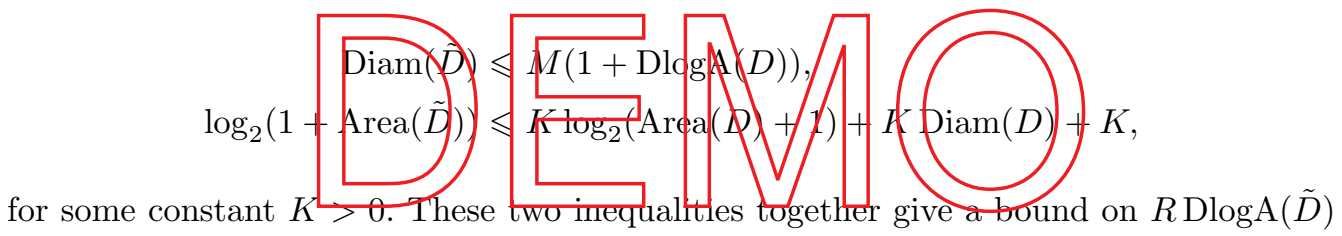
in terms of $\operatorname{Dlog} \mathrm{A}(D)$, from which $R \mathrm{D} \log \mathrm{A} \preceq \operatorname{Dlog} \mathrm{A}$ follows.

Lemma 7.3 and its dual reformulation together imply that $R$ Diam $\simeq R$ GL. The result $R$ GL $\preceq$ D $\log$ A comes from analysing our proof of GL $\preceq$ D logA (part of Theorem 7.1): we constructed a diagram $\tilde{D}$ as set out in Proposition 7.2, and all the vertices of this diagram had valences at most 12 .

For the reverse bound $\operatorname{Dlog} \mathrm{A} \preceq R$ GL we exploit (c) that tells us it is enough to show $R \operatorname{Dlog} \mathrm{A} \preceq R$ GL. The proof of this is the same as that of Proposition 7.4. This completes the proof of $(\mathrm{d})$.

That Conjecture 4.3 implies $R$ GL $\simeq R$ DGL is a direct consequence of the definitions.

The final conclusion of the theorem says that (assuming Conjecture 4.3) the restricted filling functions $R$ Diam, $R \mathrm{FL}, R \mathrm{GL}, R \mathrm{DGL}, R \mathrm{D} \log \mathrm{A}$ (as well as FL, DGL and DlogA) for a fat finite presentation are all $\simeq$-equivalent. Thus in the restricted case the universe of the filling functions we have been considering collapse to just two: $R \mathrm{FL}(\simeq \mathrm{FL})$ and $R$ Area, the restricted analogues of the (non-deterministic) space and time complexity measures, respectively, of the crude method of attacking the by word problem by exhaustively applying relators. Whether or not the non-restricted case simplifies in a similar way is open (cf. $\S 5$ of $[\mathbf{1 2}])$. 


\section{References}

1. J. M. Alonso, Inégalitiés isopérimétriques et quasi-isométries, C. R. Acad. Sci. Paris Sér. I 311 (1990), 761-764.

2. G. Baumslag, S. M. Gersten, M. Shapiro and H. Short, Automatic groups and amalgams, J. Pure Appl. Alg. 76(3) (1991), 229-316.

3. M. R. BRidson, Combings of semidirect products and 3-manifold groups, Geom. Funct. Analysis 35(3) (1993), 263-278.

4. M. R. BRIDSOn, Asymptotic cones and polynomial isoperimetric inequalities, Topology 38(3) (1999), 543-554.

5. M. R. BRIDSON, The geometry of the word problem, in Invitations to geometry and topology (ed. M. R. Bridson and S. Salamon), pp. 33-94 (Oxford University Press, 2002).

6. M. Dehn, Über unendliche diskontunuierliche Gruppen, Math. Ann. 71 (1912), 116-144.

7. R. Diestel, Graph theory, Graduate Texts in Mathematics, vol. 173, 2nd edn (Springer, 2000).

8. S. M. Gersten, Isoperimetric and isodiametric functions, in Geometric group theory I (ed. G. Niblo and M. Roller), LMS Lecture Notes, no. 182 (Cambridge University Press, 1993).

9. S. M. Gersten, Asynchronously automatic groups, in Geometric group theory (ed. R. Charney, M. Davis and M. Shapiro), pp. 121-133 (de Gruyter, 1995).

10. S. M. Gersten and T. R. Riley, Filling length in finitely presentable groups, Geom. Dedicata 92 (2002), 41-58.

11. S. M. Gersten And T. R. Riley, Filling radii of finitely presented groups, Q. J. Math. 53(1) (2002), 31-4

12. S. M. Gersten And T. R. Rleey, The gAllery lensth filling fundtipn and a geometric inequality for filling lensth, Preprin, 2003 , avallaple at http:/ w ww.math.utah.edu/ $\sim$ gersten/eprints.htm.

13. S. M. GERSTEN, D. F. HOLT hND T.R. RILEF/Is groups, Geom. Funct. Analysis 13 (2003), 795-814.

14. M. Gromov, Asymptotic invariants of infinite groups, in Geometric group theory II (ed. G. Niblo and M. Roller), LMS Lecture Notes, no. 182 (Cambridge University Press, 1993).

15. M. Gromov, Carnot-Carathéodory spaces seen from within, Progress in Mathematics, vol. 144, pp. 79-323 (Birkhäuser, 1996).

16. V. S. GuBA, The Dehn function of Richard Thompson's group $F$ is quadratic, Preprint, 2002, to appear in Invent. Math.

17. R. C. Lyndon And P. E. Schupp, Combinatorial group theory (Springer, 1977).

18. A. Yu. OL'shanskiI, Geometry of defining relations in groups (Kluwer, 1991).

19. T. R. RILEY, The geometry of groups satisfying weak almost-convexity or weak geodesiccombability conditions, J. Group Theory 5(4) (2002), 513-525.

20. T. R. Riley, Higher connectedness of asymptotic cones, Topology 42 (2003), 1289-1352. 